First Annual Progress Report on Transmission of Information by Acoustic Communication along Metal Pathways in Nuclear Facilities

Transmission of information by acoustic communication along metal pathways in nuclear facilities

FY 2017 Annual Report

Nuclear Engineering Division 


\begin{abstract}
About Argonne National Laboratory
Argonne is a U.S. Department of Energy laboratory managed by UChicago Argonne, LLC under contract DE-AC02-06CH11357. The Laboratory's main facility is outside Chicago, at 9700 South Cass Avenue, Argonne, Illinois 60439. For information about Argonne and its pioneering science and technology programs, see www.anl.gov.
\end{abstract}

\title{
Disclaimer
}

This report was prepared as an account of work sponsored by an agency of the United States Government. Neither the United States Government nor any agency thereof, nor UChicago Argonne, LLC, nor any of their employees or officers, makes any warranty, express or implied, or assumes any legal liability or responsibility for the accuracy, completeness, or usefulness of any information, apparatus, product, or process disclosed, or represents that its use would not infringe privately owned rights. Reference herein to any specific commercial product, process, or service by trade name, trademark, manufacturer, or otherwise, does not necessarily constitute or imply its endorsement, recommendation, or favoring by the United States Government or any agency thereof. The views and opinions of document authors expressed herein do not necessarily state or reflect those of the United States Government or any agency thereof, Argonne National Laboratory, or UChicago Argonne, LLC. 


\section{First Annual Progress Report on Transmission of Information by Acoustic Communication along Metal Pathways in Nuclear Facilities}

Transmission of information by acoustic communication along metal pathways in nuclear facilities

FY 2017 Progress Report

prepared by

A. Heifetz, S. Bakhtiari, X. Huang, R. Ponciroli and R.B. Vilim

Nuclear Engineering Division, Argonne National Laboratory

September 30, 2017 



\section{ABSTRACT}

The objective of this project is to develop and demonstrate methods for transmission of information in nuclear facilities by acoustic means along existing in-place metal piping infrastructure. Pipes are omnipresent in a nuclear facility, and penetrate enclosures and partitions, such as the containment building wall. In the envisioned acoustic communication (AC) system, packets of information will be transmitted as guided acoustic waves along pipes. Performance of $\mathrm{AC}$ hardware and network protocols for efficient and secure communications under development in this project will be eventually evaluated in a representative nuclear power plant environment.

Research efforts in the first year of this project have been focused on identification of appropriate transducers, and evaluation of their performance for information transmission along nuclear-grade metallic pipes. COMSOL computer simulations were performed to study acoustic wave generation, propagation, and attenuation on pipes. An experimental benchtop system was used to evaluate signal attenuation and spectral dispersion using piezo-electric transducers (PZTs) and electromagnetic acoustic transducers (EMATs). Communication protocols under evaluation consisted on-off keying (OOK) signal modulation, in particular amplitude shift keying (ASK) and phase shift keying (PSK). Tradeoffs between signal power and communication data rate were considered for ASK and PSK coding schemes. 


\section{TABLE OF CONTENTS}

ABSTRACT …

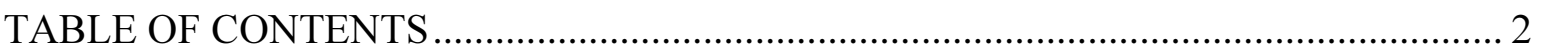

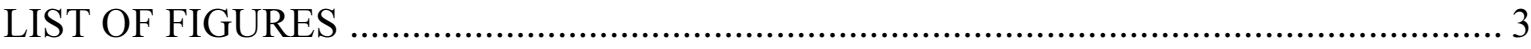

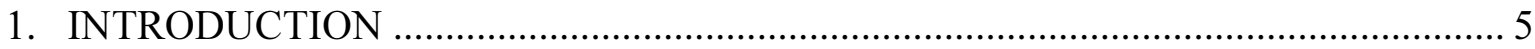

1.1. Aim of the Project and Background ................................................................. 5

1.2. Evaluation of potential means for nuclear unit communication technologies......... 6

2. IDENTIFICATION OF ACOUSTIC PATHWAYS …………………..................... 8

2.1. General Information on NPP Containment Penetrations......................................... 8

2.2. Specific Piping Systems of Interest .................................................................. 10

2.3. Characteristics of Identified Piping System ………........................................... 11

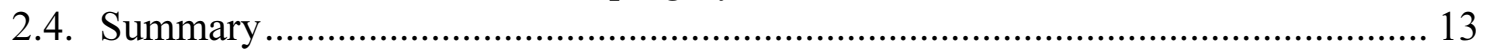

3. MODELING ULTRASONIC WAVES IN PIPE-LIKE STRUCTURES ...................... 13

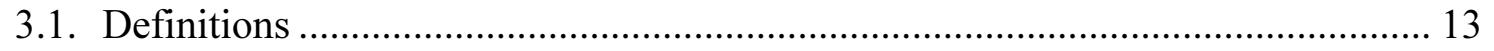

3.2. Selection of Ultrasonic Modes.......................................................................... 14

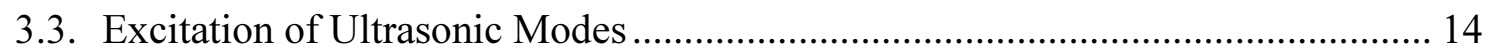

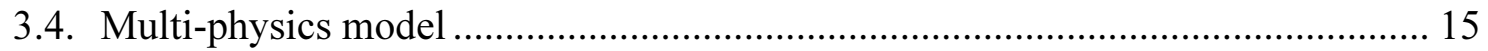

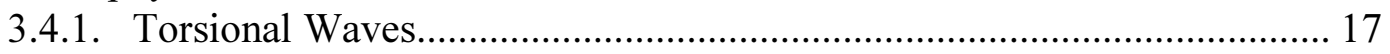

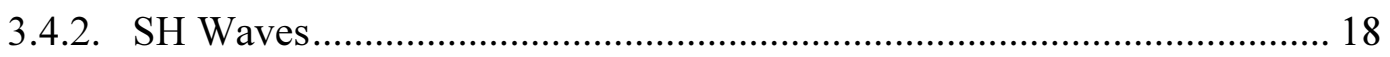

3.4.3. Non-axisymmetric Waves...................................................................... 20

3.4.4. Influence of Elbow on Ultrasonic Wave Propagation................................ 20

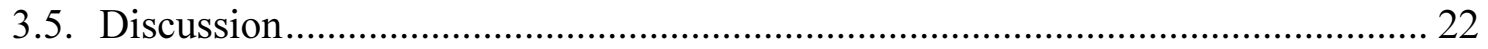

4. ULTRASONIC COMMUNICATION SYSTEM..................................................... 22

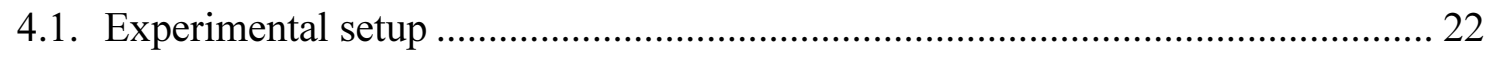

4.1.1. Pipe

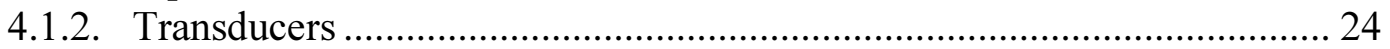

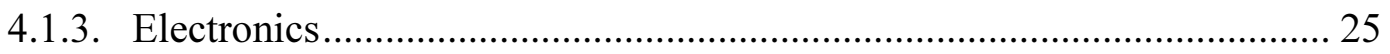

4.1.4. Data acquisition............................................................................... 25

4.2. Evaluation of modulation methods in PZT-based experimental setup ................... 26

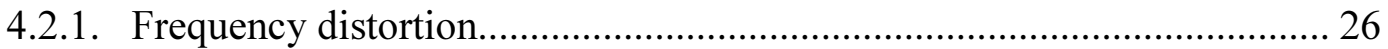

4.2.2. OOK modulation................................................................................ 27

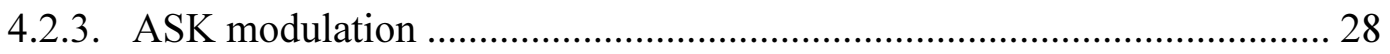

4.2.4. PSK modulation .................................................................................. 29

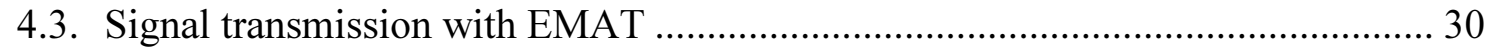

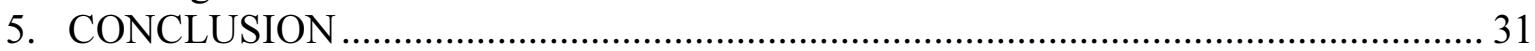

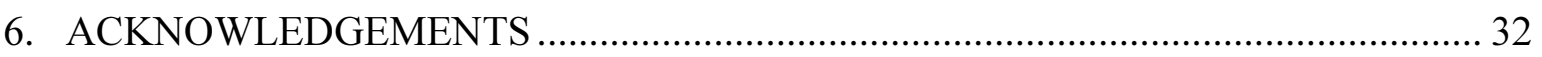

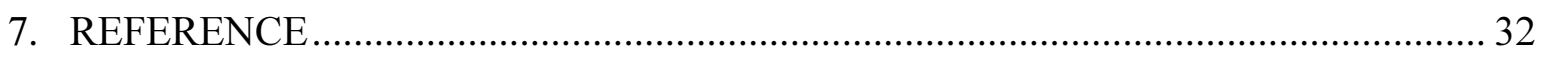




\section{LIST OF FIGURES}

Figure 1. Interior view of Prototype Generation IV Reactor, showing the extensive in-situ piping network, (b) schematic drawing of proposed communication concept......................................... 5 Figure 2. Schematic drawing of refracted acoustic wave transmission and reception using

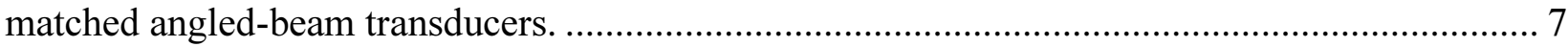

Figure 3. Chemical and Volume Control System Schematic [25] ........................................... 10

Figure 4. Typical Double Barrier Protection for Piping through Containment. ............................. 12

Figure 5. Dispersion curve of 3-inch schedule 40 steel pipe [31] ............................................. 14

Figure 6. The different sensor configurations for excitation of ultrasonic wave modes ([31][36]).

Figure 7. Excitation signals, (a) chirp signal, (b) single frequency signal.................................... 16

Figure 8. Chirp signals in the range of $30 \mathrm{kHz}-100 \mathrm{kHz}$, (a) convex mode, (b) linear mode..... 16

Figure 9. Assessment of numerical model with [31] ............................................................ 16

Figure 10. Pipe model and excitation of torsional wave mode................................................... 17

Figure 11. Responses of circumferential displacement at different locations, (a) under convex

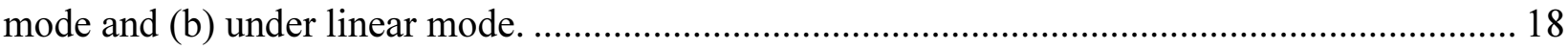

Figure 12. (a) EMATs design, (b) EMATs simplified model...................................................... 18

Figure 13. Response signals due to axial SH wave mode......................................................... 19

Figure 14. Circumferential SH wave propagation modeling. ...................................................... 19

Figure 15. Response signal due to circumferential SH wave mode........................................... 20

Figure 16. Response signal in non-axisymmetric wave mode..................................................... 20

Figure 17. (a) The model of pipeline with elbow, (b) wave group propagation near the elbow. .. 21

Figure 18. Response signals of the pipeline model with elbow, (a) time domain (b) frequency

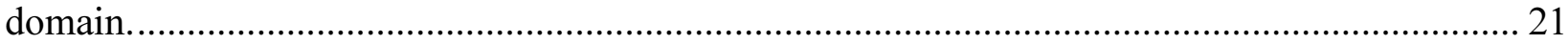

Figure 19. Windowed response signals, (a) time domain (b) frequency domain. ........................ 22 Figure 20. Schematic of the laboratory system for evaluation of acoustic communication along

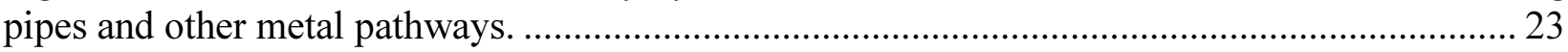
Figure 21. Photo of the laboratory setup showing various components of the acoustic communication system. Displayed at the bottom is the pipe mounted with angled transducers... 23 Figure 22. Snell's Law of angle beam transducer. ........................................................................ 24

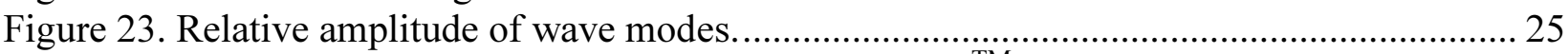
Figure 24. Virtual instrument developed under $\mathrm{LabView}^{\mathrm{TM}}$ to generate, detect and analyze

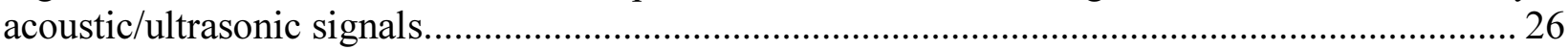
Figure 25. Spectral dispersion and distortion of narrow band pulses characterized by different

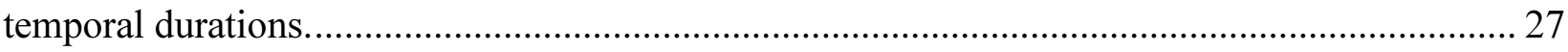

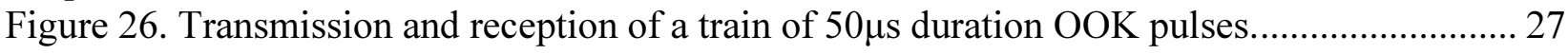

Figure 27. Transmission and reception of a train of $100 \mu$ s duration OOK pulses......................... 28

Figure 28. Example of ASK modulation with $50 \mu$ s bit pulse duration. Transmitted and received signals are overlaid with amplitudes not to scale .......................................................................... 28 Figure 29. Examples of ASK transmitted signals (on the left) and received signals (on the right). Top panel: binary signal "11001011". Bottom panel: binary signal "1101011"............................ 29

Figure 30. Example of PSK signal with 50 micro-s bit pulse duration......................................... 29 Figure 31. Example of PSK modulation, (a) binary signal "1100", (b) binary signal "1001"....... 30 Figure 32. (a) EMAT transmitted signal spectrum, (b) PZT received signal spectrum.................. 31 Figure 33. Example of time-domain waveform transmitted from EMAT to PZT.......................... 31 


\section{LIST OF TABLES}

Table 1. Description of Typical Westinghouse PWR Penetrations. ............................................ 9

Table 2. Pipe dimensions and materials properties (DN65-SCH160) ..................................... 15 


\section{INTRODUCTION}

\subsection{Aim of the Project and Background}

This project aims to develop and demonstrate methods for transmission of information in nuclear facilities by acoustic means along existing in-place metal pipes. This innovative means of transmitting information overcomes physical hurdles that beset conventional communication methods, i.e. both wired and radio frequency (RF) wireless. This technology is intended for those nuclear facilities where wired or wireless RF communication is not feasible (presence of barriers), not reliable (lack of resilience under accident conditions), or not secure (prone to interception). Use of wireless RF signals for the transmission and reception of data in nuclear facilities provides, in principle, greater data transfer rate per unit cost. Unfortunately, the presence of physical boundaries presents a major challenge to its actual implementation. The typical nuclear facility for safety reasons is heavily partitioned and equipment-packed resulting in transmission paths that are highly attenuating for electromagnetic waves. Primary barriers include a containment building's thick reinforced high-strength concrete walls, which in some plant designs have liners (steel plates) on the interior side. Additional security-related concerns related to use of $\mathrm{RF}$ exist because of long distance propagation of RF signal outside of nuclear facility boundaries.

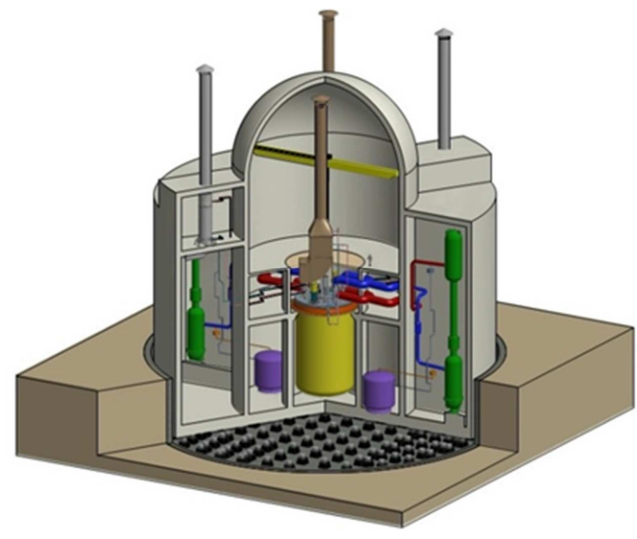

(a)

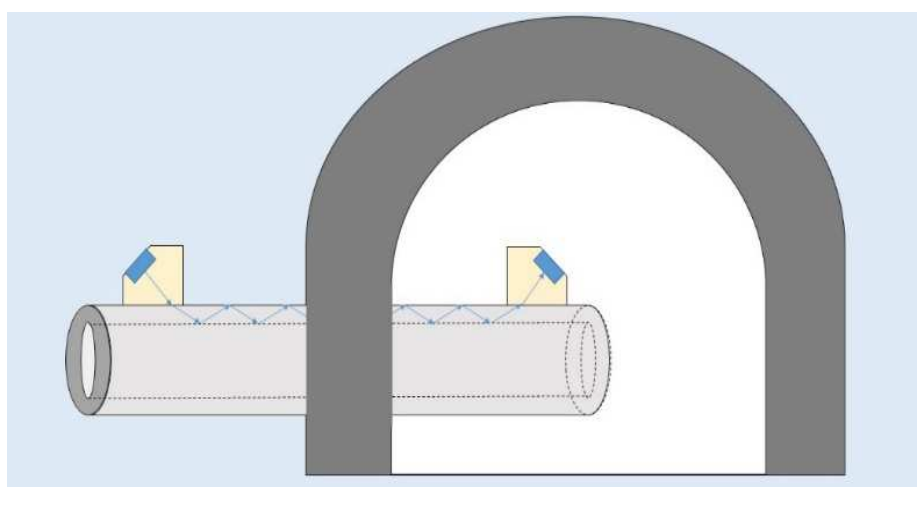

(b)

Figure 1. Interior view of Prototype Generation IV Reactor, showing the extensive in-situ piping network, (b) schematic drawing of proposed communication concept.

From this standpoint, the natural piping infrastructure of a facility provides, however, an alternate means for dealing with these communication barriers. Pipes are omnipresent in a nuclear facility given their role of transferring mass and energy between the outside world and the inner workings of the facility. For example, in a commercial nuclear plant, pipes carry cooling water to the reactor vessel and spent fuel pool, transport steam to the main turbine, provide hydrogen gas to cool the main generators, supply fuel and lubricating oil to the emergency diesel generators, and supply water to fire-extinguishing sprinklers [1]. Process sensors, such as temperature, pressure and flow meters are located on pipes as well. Therefore, these metal pathways penetrate all sectors of the plant by providing a natural conduit for information transfer through physical barriers.

The aim of this work is the development of acoustic communication (AC) hardware and network protocols for efficient and secure transfer of data, and to provide a preliminary experimental demonstration of such an $\mathrm{AC}$ system. By adopting pipes and other metal structures as conduits for 
signals launched as guided acoustic surface waves, the proposed acoustic system represents a low-cost, energy-efficient communication option, which is compatible with RF wireless communication due to availability of commercial acoustic to RF transducers.

\subsection{Evaluation of potential means for nuclear unit communication technologies}

While RF wireless communication technology has found widespread commercial use, its utility and practicality in a nuclear facility has drawbacks. Establishing a wireless RF system at a nuclear facility requires careful positioning of transmitters and receivers that take into account the plant topology [2]. In case of a disruptive event, such as a major natural disaster or accident, wireless sensors are likely to be perturbed, and thus the communication network in covered areas might be compromised. Another challenge for, post-accident conditions operations is that water sprays from fire-extinguishing sprinklers could strongly scatter free-space RF waves and damage sensitive electronic equipment. In any case, the main issue which challenges the use of RF communication systems is the presence of physical boundaries. In another work, the propagation of electromagnetic waves through reinforced concrete was studied. In principle, RF transmission through reinforced concrete walls without liners is possible, but would need to be performed at frequencies substantially lower than those of commercially operating $900 \mathrm{MHz}$ and $2.4 \mathrm{GHz} \mathrm{RF}$ wireless systems. Prior studies have shown RF in $100 \mathrm{MHz}$ to $200 \mathrm{MHz}$ (VHF) frequency band transmission through three foot-thick wall with approximately $20 \mathrm{~dB}$ attenuation [3]. RF attenuation was shown to rise with increasing RF frequency, e.g. for $900 \mathrm{MHz}$ frequency, attenuation is approximately $40 \mathrm{~dB}$, and for $2.4 \mathrm{GHz}$, attenuation is over $50 \mathrm{~dB}$. Therefore, using commercial frequency standards for through-concrete transmission would not be practical because such system would require using power amplifiers, which are energy consuming. In postaccident operation conditions, energy consumption by amplifiers would be a big burden.

One could envision developing through-wall RF communication system operating at VHF frequency, with transducers coupling to higher frequency (currently $2.4 \mathrm{GHz}$ ) inside the facility. However, a significant concern is that broadcasting through facility walls makes RF wireless networks vulnerable to eavesdropping by unauthorized users, even at a substantial distance (potentially about a kilometer) away from a nuclear power plant. This is because RF antennas for wireless communications often have omnidirectional radiating spatial patterns, and because of the potential for long distance propagation of RF signals outside of the nuclear complex, which can be picked up by sensitive RF receivers. Because of this exposure, even with an encryption layer in place, wireless networks could be vulnerable to sophisticated attacks.

Similarly, transmission through facility walls by acoustic waves launched directly into concrete is impractical as well because of the large signal losses, i.e. high-frequency ultrasonic waves (frequency above $20 \mathrm{kHz}$ ) suffer extinction losses on the order of approximately100 dB/m [4]. Besides extinction losses, a challenge is to couple ultrasound waves into concrete because of losses caused by impedance mismatch. Existing literature on nondestructive testing (NDT) studies of reinforced concrete structures indicates that four-foot thick reinforced concrete walls (typical thickness of containment structure) admit extremely low acoustic frequencies (below 20 $\mathrm{Hz}$ ). Such low acoustic bandwidth would make data transmission at meaningful rates impossible, since even at extremely high spectral efficiencies of up to $10 \mathrm{bits} / \mathrm{sec} / \mathrm{Hz}$ (2-4 would be more likely at modest signal to noise ratios), less than 200 bits per second would be possible, with 20 bits per second more likely. 
Because of these issues and limitations, an alternate pathway which involves the transmission of guided acoustic waves over the metal structures that typically penetrate concrete barriers is proposed. In this way, an additional level of protection for securing and protecting data streams by eliminating the broadcasting of RF signals outside the facility is provided. There is a long history of using guided acoustic waves in NDT of metallic and ceramic structures and components. Acoustic waves could be excited with inexpensive impedance-matched transducers.

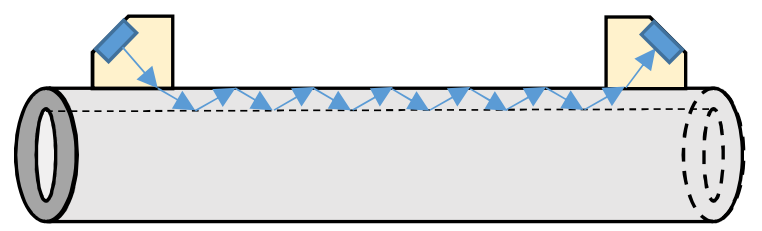

Figure 2. Schematic drawing of refracted acoustic wave transmission and reception using matched angled-beam transducers.

Figure 2 displays a schematic drawing of an acoustic wave transmission along a pipe using a pair of matched transducers. Angled beam transducers and wedges are typically used to introduce a refracted shear wave into material for NDT applications. The same approach could be utilized for acoustic information transmission over pipes. Acoustic communication over pipes has been recently investigated by several research groups. Their efforts were aimed primarily towards developing communication systems for pipeline networks for the oil and gas industry; however, recent interest in other pipe infrastructure has emerged [5]. Recently filed IP disclosures included general concepts [6], hybrid RF and AC systems [7], and communication for geological survey downhole instrumentation [8]. Some prior peer-reviewed journal studies of AC schemes targeted low data rates ( 100bps), but focused on the choice of modulation/demodulation. For example, suggested simple on/off keying (OOK) modulation for ultrasonic communication along metal pipes; [9] proposed time-reversal position modulation (TRPPM); [10] used chirp on-off keying (C-OOK) to mitigate frequency selectivity of the acoustic channel along metal pipes; [11] proposed to use cyclic frequency shifting.

Recent research indicates that emerging $\mathrm{AC}$ technology holds enormous promise for secure and accident-resilient communication in severe environments. In comparison to RF, acoustic channels typically have lower communication bandwidth. However, recent advances in digital signal processing for underwater acoustic communications have shown the possibility of efficient exploitation of acoustic bandwidth for transmission of large amounts of data [12-18]. Note that acoustic networks can be integrated with commercial RF wireless networks, since two-way acoustic to RF transducers are being commercially developed, such as those described in [18] targeting an Ethernet interface to an acoustic modem. For example, once communication in and out of the containment building using acoustic signals is established, directional RF wireless routers can be placed along the piping backbone to set up multiple wireless local area networks (LANs). From this standpoint, the proposed AC technology is complementary to RF wireless development by telecommunication industry. 


\section{IDENTIFICATION OF ACOUSTIC PATHWAYS}

As first step, the candidate metallic pathways that hold promise for transmission of information by acoustic means need to be identified. Therefore, a review of the metal piping infrastructure in a commercial nuclear power plant (NPP) is performed. In addition, the locations for the transmission of accident monitoring instrumentation (AMI) data need to be identified.

While it would first appear that there are many opportunities in a typical Light Water Reactor (LWR) to exploit existing containment penetrations to move information through piping, this is not quite the case. There are several constraints that need to be accounted for. The types of penetration, in terms of size, piping and insulation material, and the operating conditions are to be identified. In particular, the piping material affects the dispersion of acoustic signals. Operating conditions in terms of flow and pipe temperatures are relevant as well, and so is the specific role of each piping penetration under investigation (safety-related components are more difficult to be accessed compared to routinely used penetrations). Also, containment penetrations may be isolated during accidental conditions through the use of isolation valves, with may impact on acoustic wave reflection, dispersion and signal noise. Finally, how the presence of flowing fluid inside the pipe affects the acoustic transmission properties must be determined too, by accounting for all the conditions that a given penetration may be subjected to during both operational and accidental scenarios.

Given such a large and complex investigation space it is necessary to first perform a downselection of piping penetrations to identify the most promising option. In order to perform such selection, a top-to-bottom approach is here followed. In Section 2.1 general information on NPP containment penetration is reviewed, followed in Section 2.2 by the identification of a few specific piping systems of interest. Finally Section 2.3 lists the characteristics of the piping system identified as the most promising one.

\subsection{General Information on NPP Containment Penetrations}

In an LWR, three types of containment penetrations can be identified [19]: piping penetrations, electrical penetrations [20] and air locks, door and hatches [21]. In terms of size, containment penetrations fall into two major categories, large and small. Large penetrations are defined as those having an inside diameter equal to or greater than 10 feet (2.5 times the nominal shell wall thickness). The equipment hatch and the personnel hatch fall into this category. Small penetrations include miscellaneous piping, electrical, and ventilation penetrations. Typically, all containment penetrations are designed for the same loads and load combinations as the containment structure, and for the forces stemming from pipe movements or accidental loads. For the transmission of acoustic signals, clearly only piping penetrations are of interest and therefore only such type of penetration is here considered. It should be noted that piping penetrations should be accessible so that leaks from individual penetrations can be detected in the leak tightness tests. Such criterion of accessibility is an additional feature compatible with the installation and inspection of devices for transmission of acoustic signals through piping penetrations.

The US Nuclear Regulatory Commission (NRC) requires that piping systems penetrating containment can be isolated (10CFR50, App. A, GDC 54). The containment isolation functions to 
isolate the containment from the outside environment in the event of any postulated accident for which isolation is required. Design criteria for this function are contained in General Design Criteria (GDC) 54 through 57, Regulatory Guide 1.141, NUREG-0737, and Section 6.2.4 of the Standard Review Plan. Therefore, containment penetrations are typically associated with containment isolation systems, which allow the normal or emergency passage of fluids through the containment boundary while minimizing the release of fission products-from containment following an accidental scenario triggering the containment isolation activation [22][23].

In particular, reactor coolant pressure boundaries penetrating containment (10CFR50, App. A, GDC 55) must isolable according to one of the following solutions:

- One locked closed isolation valve inside and outside containment,

- One locked closed isolation valve inside and one automatic valve outside containment,

- One automatic valve-or check valve inside-and one-locked closed isolation -valve outside containment, or

- One automatic valve or check valve inside and one automatic valve outside containment

In Pressurized Water Reactors (PWRs) [22], there are three isolation phases:

- Phase A: containment phase A isolation (CISA) signal serves to isolate containment in the event of a loss of coolant accident or steam break. This signal actuates closure of all valves that are not required to be open for the operation of essential equipment. A CISA actuation signal originates with a safety injection or manual actuation.

- Phase B: containment phase B isolation (CISB) signal serves to isolate containment on a steam break or-loss of coolant accident. It isolates penetrations that were not isolated by the CISA signal and which are not required for operation of the engineered safety features equipment. In particular, this isolates component cooling water to the reactor coolant pumps.

- Containment Purge: containment purge isolation system (CPIS) detects any abnormal amount of radioactivity in the containment atmosphere or in the containment purge effluent and initiates appropriate action to ensure that any release of radioactivity to the environment is controlled. This appropriate action is shutting the purge system isolation valves and deenergizing the purge system fans. The CPIS protects against activity release following a loss of coolant accident or fuel handling accident.

For this investigation, it is of interest to identify in particular routinely used penetrations (including related valves and systems) for LWRs (and in particular PWRs). Typical Westinghouse PWR penetrations are reported in Table 1 [23].

Table 1. Description of Typical Westinghouse PWR Penetrations.

[Table withheld. Please contact the author]

Among the sixteen penetrations listed in Table 1, the most promising for consistent acoustic signals transmission are to be identified. It should be kept it mind that consistent data transmission is likely to be associated not with the largest penetrations or with penetrations having very specific roles during accidental conditions. Instead it is expected that piping penetrations characterized by steady operating conditions that are possibly isolated during 
accidental scenarios may offer ideal conditions for acoustic signal transmission. In the next section specific piping systems of interest will be analyzed in greater detail.

\subsection{Specific Piping Systems of Interest}

Looking at the list of PWR containment penetration of Table 1, a few piping systems of interest can be identified. In particular steam and feed-water lines, emergency systems (such as safety injections and residual heat removal systems) and auxiliary systems (such as the CVCS, chemical and volume control system). However, taking into account the general requirements discussed in previous sections such as low temperature, steam and feed-water lines cannot be considered promising options. In addition, penetrations associated to emergency systems are necessarily safety-grade components and it would be excessively burdensome, from a regulatory point of view, to implement an acoustic communication system on such lines. As a consequence, only penetrations associated with auxiliary systems are left as viable options. In particular, among auxiliary systems that are not site-specific, the CVCS meets several of the desired criteria. Detailed description of PWR CVCS can be found in [24], [25], and [26]. The most relevant elements for this investigation are summarized in Section 2.4.

As described in [24], the Chemical and Volume Control System (CVCS) is designed to perform the following functions in support of the Reactor Coolant System (RCS).

- Maintains required water inventory in the RCS by maintaining programmed water level in the pressurizer

- Reduces the concentration of corrosion and fission products in the reactor coolant

- Provides a means for filling, draining, and pressure testing the RCS

- In conjunction with the Boron Thermal Regeneration System (BTRS), adjusts the boric acid concentration of the reactor coolant for chemical shim control

- Provides a means for control of RCS chemistry

- Provides high-pressure seal water for the reactor coolant pump (RCP) seals

In addition and with particular relevance to this work, it should be taken into account that as described in [25], several changes occur in the chemical and volume control system when an engineered safety features actuation signal is received. These changes are listed below:

1. Letdown is isolated by closing the letdown containment isolation valves.

2. The normal charging path is isolated by closing the redundant charging line isolation valves.

3. The suction to the charging pump is transferred from the volume control tank to the refueling water storage tank by closing the series volume control tank outlet valves and opening the parallel refueling water storage supply valves.

4. Both centrifugal charging pumps are started.

The centrifugal charging (high head safety injection) pumps discharge through isolation valves which open upon an engineered safety features actuation signal.

\section{[Graphic image withheld. Please contact the author]}

Figure 3. Chemical and Volume Control System Schematic [25]. 


\subsection{Characteristics of Identified Piping System}

Upon inspection of the CVCS schematics and the related containment penetrations, only the charging line emerges as a promising location for the application of an acoustic transmission device. This is because only the charging line offers the combination of two favorable characteristics: a relatively low temperature and containment isolation valves both placed outside containment. In terms of normal operating conditions, relevant information appears in [27]. In particular with respect to the charging line, the following data are relevant and are representative of the water conditions in the charging line at the containment penetration during normal, steady state operating conditions:

- Water mass flow rate: 3.45 to $6.3 \mathrm{~kg} / \mathrm{s}$ (55 to100 gpm)

- Water temperature: $55^{\circ} \mathrm{C}$

- Design pressure: $\quad 21.37 \mathrm{MPa}(17 \mathrm{MPa}$ estimated operating pressure)

- Pipe nominal dia.: 5.08 to $7.62 \mathrm{~cm}$ (2 to 3 inches)

In terms of materials, in [27] it is stated that all CVCS piping that handles radioactive liquid (such as the charging line) is made of austenitic stainless steel. In addition, all piping joints and connections are welded except where flanged connections are required to facilitate equipment removal for maintenance and hydrostatic testing. In addition, in [28] information about the type of insulation and insulation material used for the CVCS charging lines inside containment can be found. For example, in the Calvert Cliffs units the insulation for the charging line is encapsulated. The actual insulation material is mineral wool in panels. The panels typically consist of 22 gauge stainless steel sheet, a thickness of 9 pound density mineral wool (corresponding to about 2 inch thickness), and a 24 gauge stainless steel sheet. For other plants, calcium silicate is reported as insulation material for the encapsulated insulation for CVCS piping. In particular, calcium silicate molded blocks are provided in thicknesses up to 4 inches and lengths of up to 3 feet. For such blocks then the jacketing used may be fiberglass cloth, stainless steel or aluminum.

While the survey of insulation materials used in NPP reported in [28] is useful for identifying typical materials and dimensions, more recent plants may adopt different insulation materials or configurations. An ongoing effort is focused on accessing more recent source of information and on inquiring reactor vendors directly as well.

Typical PWR containment building are made of concrete walls that are about 4 feet $(1.2 \mathrm{~m})$ thick and have typically an inner steel liner plate (nominally 6 to $10 \mathrm{~mm}$ ) in contact with the concrete shell. Containment penetrations can be therefore quite complex given the amount of structure to go through and in addition the presence of insulation valves can further complicate the layout of piping around a penetration both on the inside and on the outside of the containment structure itself.

A review of the containment penetrations and isolation systems for a Westinghouse PWR can be found in [22]. 


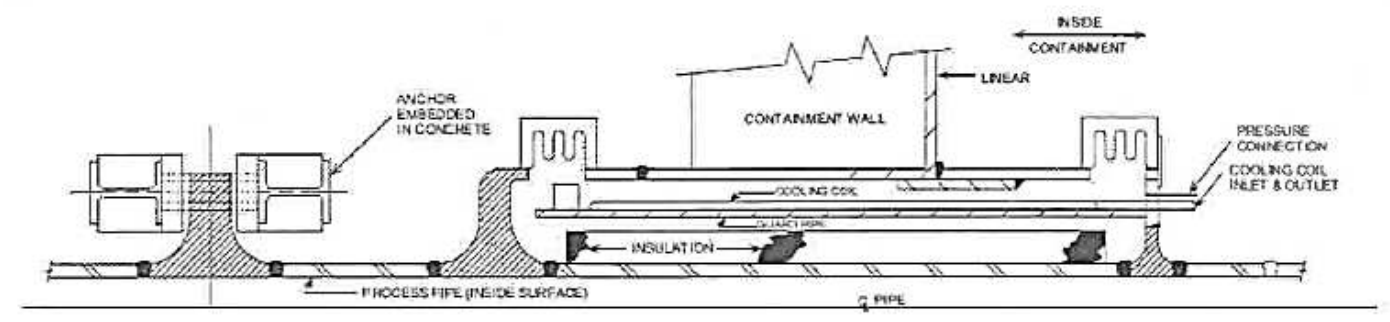

MAINSIEAM S FEED WATER PENETRATIONASSEMBIY

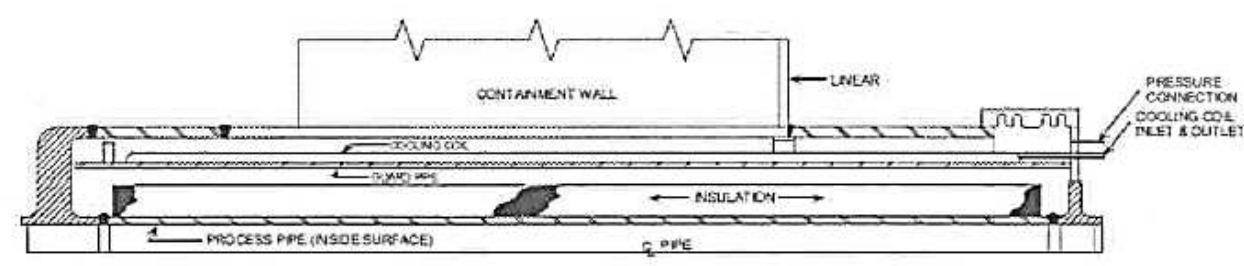

TYPICAL HOT PENETRATION ASSEMBLY

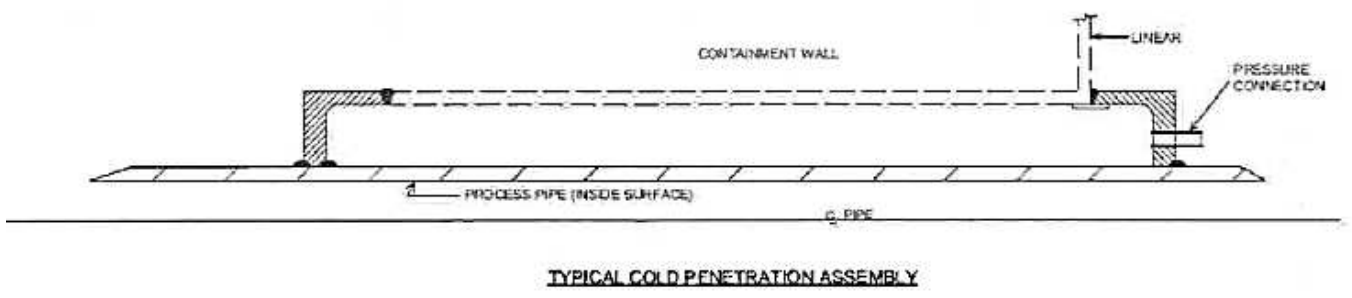

Figure 4. Typical Double Barrier Protection for Piping through Containment.

In particular, quoting directly from [22], double barrier piping penetrations are provided for all piping which passes through the containment. The pipe is contained in a sleeve which is welded to the liner. Closure heads are welded to the sleeve and to the pipe, both inside and outside the containment, to form the double barriers. The annulus between the pipe and sleeve is continuously pressurized. Several pipes may pass through the same sleeve to minimize the number of containment penetrations required. In these cases, each pipe is welded to both closure heads. The penetrations for main steam, feedwater, and steam generator blowdown are anchored outside the containment in such a way as to ensure containment integrity should any one of these lines rupture. These penetrations are provided with a bellows expansion joint on both the inside and the outside of the containment, which allows for differential movement between the containment wall and the anchor. The inside joint will also take up the differential movement of the hot pipe relative to the liner. The expansion joints have been designed so that, in the event of pipe ruptures within the sleeve, the inside joint will subsequently rupture and the outside joint will remain intact, thereby maintaining containment integrity. Hot penetrations are normally provided with some type of cooling to minimize drying of the containment concrete around the penetrations. Other hot penetrations, which are not anchored outside the containment for pipe rupture, are where required, provided with a single expansion joint, either inside or outside the containment, to allow for thermal movement of the pipe relative to the anchor. 
The CVCS charging line penetration does either qualify as a hot or cold penetration, depending on whether the piping insulation is carried through containment. This aspect is currently being investigated to further characterize the actual penetration configuration and conditions.

Finally it is of interest to point out that the CVCS charging line is isolated when an engineering safety features signal is received as pointed out in [25]. Isolation valves are placed outside containment and therefore do not impact locally the actual containment penetration which is to be considered a promising location for installing acoustic transmission devices. The fact that the charging line is to be isolated during accidental conditions means that in such scenarios no water would be flowing through. However, this does not necessarily imply that the line is also drained and therefore no stagnant water is left. As a consequence, the actual conditions during accidental scenarios cannot be defined a priori and acoustic transmission devices to be applied to the CVCS charging line across the containment penetration should be over full range of conditions from empty/full stagnant to full flowing water pipes.

\subsection{Summary}

The findings presented in Section 2 are summarized:

- Most promising system in PWRs: CVCS

- Most promising piping and location: Charging Line - Containment Penetration

- Piping material: Austenitic Stainless Steel

- Typical insulating materials: Mineral Wool-Calcium Silicate

- Typical Insulation thickness: $2-4$ inches $=\mathbf{5 . 0 8}-\mathbf{1 0 . 1 6} \mathbf{~ c m}$

- Typical water mass flow rate $=55-100 \mathrm{gpm}=\mathbf{3 . 4 5}-\mathbf{6 . 3} \mathbf{~ k g} / \mathbf{s}$

- Typical water temperature $=\mathbf{5 5}^{\circ} \mathbf{C}$

- $\quad$ Typical line design pressure $=\mathbf{2 1 . 3 7}$ MPa (17 MPa estimated operating pressure)

- Typical piping nominal diameter $=2-3$ inches $=\mathbf{5 . 0 8}-\mathbf{7 . 6 2} \mathbf{~} \mathbf{~ m}$

\section{MODELING ULTRASONIC WAVES IN PIPE-LIKE STRUCTURES}

Once identified the piping element which is suitable to be used as an acoustic communication medium, the wave propagation characteristics of ultrasonic waves in pipe-like structures for proper selection of frequency and wave mode to increase the transmission distance of information through metal pathway needs to be studied. There are numerous guided wave modes in a pipe, which include rotational and axially symmetric torsional waves $(T(0, m)$ ), axially symmetric longitudinal waves $(L(0, m))$, and non-axially symmetric flexural waves $(L(n, m))$, and can be triggered by controlling the position, vibration direction and phase of excitation element [29]. The first index implies the harmonic order of circumferential variation, and zero means axially symmetric. The value 1 represents the fundamental modes, and higher order modes are numbered consecutively [30]. The basic factors of mode selection in this study are dispersion, attenuation, and excitability.

\subsection{Definitions}

The following definitions for terms that appear in Section 3 are provided for the reader and were collected from the World Wide Web. 
Elastic wave - A wave in which the propagated disturbance is an elastic deformation of the medium.

Longitudinal wave - A wave that propagates in the same direction as the displacement of the transmitting medium.

Torsional wave - A wave of flexure in which the elements of the medium carrying the wave perform torsional oscillations about an axis parallel to the direction of propagation.

Lamb wave - A wave whose particle motion lies in the plane that contains the direction of wave propagation and the plane normal.

Burst signal - A relatively high-bandwidth transmission over a short period of time.

Chirp signal - A signal that sweeps linearly from a low to a high frequency

\subsection{Selection of Ultrasonic Modes}

Dispersion is defined as frequency-dependent wave velocity. Depending on the selected wave mode, the ultrasonic signal may be distorted along propagation distance. In Figure 5 the dispersion curves of a 3 -inch, schedule 40 steel pipe are shown. Typically, symmetric wave modes are less dispersive. For instance, the fundamental torsional mode $T(0,1)$ has the same phase velocity for a range of frequencies while higher order non-symmetric torsional modes have different phase velocities especially in the range of frequencies lower than $300 \mathrm{kHz}$, which causes significant distortion in wave with distance. A proper transmission of signal through pipe material requires selection of non-dispersive wave modes.

\section{[Graphic image withheld. Please contact the author]}

Figure 5. Dispersion curve of 3-inch schedule 40 steel pipe [31].

\subsection{Excitation of Ultrasonic Modes}

The excitation of a particular wave mode is achieved by predetermined phase velocity and frequency to excite a point on the dispersion curve of a specific guided wave mode. In some cases, the traditional bulk wave can be used for generating waves in pipes [32]. However, angle beam method, comb array and EMATs are shown as the most efficient methods in terms of mode selection and control [33][34]. The comparison of different sensor configurations is illustrated in Figure 6. Though the sensor type and configuration are different, the core idea is the same, which is to control and select proper wave mode and frequency. The excitation of axisymmetric modes requires axisymmetric loading, which can be done by axisymmetric placement of transducers. For instance, if all the transducers in a ring positioned on the circumference of pipe are excited simultaneously, an axially symmetric mode is triggered [35]. 


\section{[Graphic image withheld. Please contact the author]}

Figure 6. The different sensor configurations for excitation of ultrasonic wave modes ([31][36]).

\subsection{Multi-physics model}

A multi-physics model was developed to investigate the characteristics of acoustic/ultrasonic wave propagation in pipe-like structures. Such models allow the parameter space to be investigated more thoroughly compared to laboratory experiments yielding better insight into performance limits of acoustic signal transmission over complex geometries, including dispersion, absorption and scattering in different metallic media. Modeling tools can further help optimize the design parameters in a more cost effective manner. The commercially available multi-physics software COMSOL ${ }^{\mathrm{TM}}$ [37] was selected for modeling efforts in this work. The ultimate goal of this activity is to increase the acoustic transmission distance by proper selection of operating frequency and excitation mode.

The pipe properties that most influence the ultrasonic wave propagation are thickness, diameter, material properties, surrounding and internal materials, pipe elbows and the presence of dissimilar materials. In Table 2, the properties selected for the finite element numerical models are shown.

Table 2. Pipe dimensions and materials properties (DN65-SCH160).

\begin{tabular}{cccccc}
\hline $\begin{array}{c}\text { Length } \\
(\mathrm{cm})\end{array}$ & $\begin{array}{c}\text { Outer Diameter } \\
(\mathrm{mm})\end{array}$ & $\begin{array}{c}\text { Wall Thickness } \\
(\mathrm{mm})\end{array}$ & $\begin{array}{c}\text { Young's Modulus } \\
(\mathrm{GPa})\end{array}$ & $\begin{array}{c}\text { Density } \\
\left(\mathrm{kg} / \mathrm{m}^{3}\right)\end{array}$ & $\begin{array}{c}\text { Poisson's } \\
\text { ratio }\end{array}$ \\
\hline 121.92 & 73.0 & 9.525 & 200 & 8000 & 0.27 \\
\hline
\end{tabular}

The spatial and temporal discretization of wave propagation problem should be selected such that wave distortion due to numerical error should be minimized. The discretization is selected as linear displacement field, which can not only ensure the accuracy but also reduce the computational time. The pipe model has the mesh size of $2.5 \mathrm{~mm}$ using tetrahedral elements. The time step is selected as $1 \mu \mathrm{s}$, which corresponds to $200 \mathrm{kHz}$ frequency resolution. The excitation signal is selected as a chirp signal with different frequency windows as well as a single excitation signal. Figure 7a shows the chirp signal in the frequency range of 100-200 kHz, and Figure $7 \mathrm{~b}$ shows the single frequency signal at $5 \mathrm{kHz}$. When the excitation frequency decreases, its duration increases such that the interference of propagating waves and reflected waves increases. 


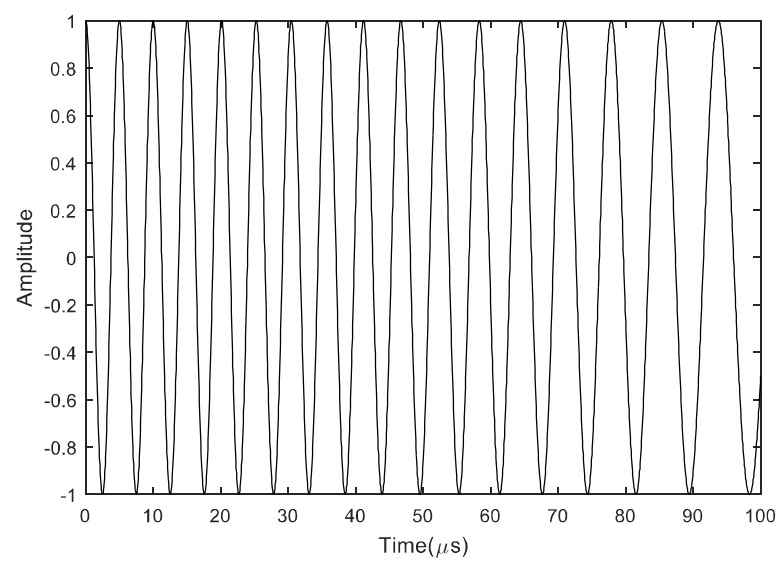

(a)

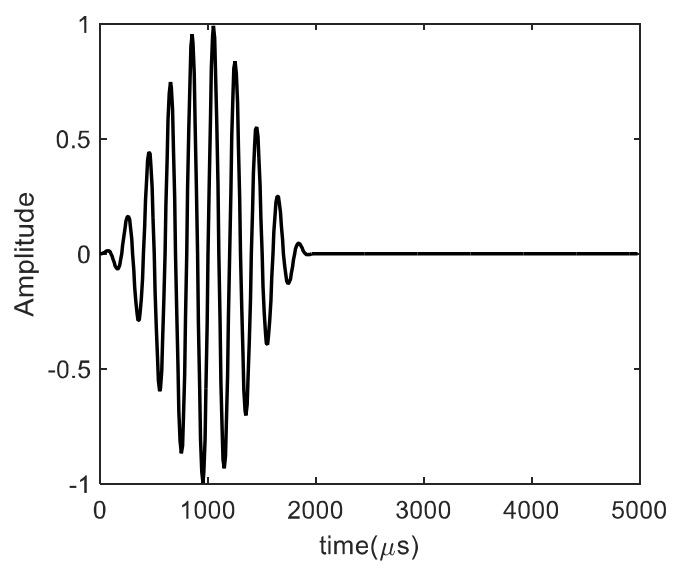

(b)

Figure 7. Excitation signals, (a) chirp signal, (b) single frequency signal.

In general, the resolution of the chirp signal depends on the duration and sampling frequency. The chirp signal is generated in both quadratic convex and linear modes in this study. Two chirp modes describe two kinds of curves in the positive frequency axis. The quadratic convex model is down-sweep, and the curve between the maximum and minimum frequency is quadratic parabola while linear chirp fades linearly from low to high frequency components. Though the quadratic convex and linear chirp signals are designed with the same frequency bandwidth, duration and sampling frequency, the instantaneous frequency varies linearly in linear mode while nonlinearly in quadratic convex mode.

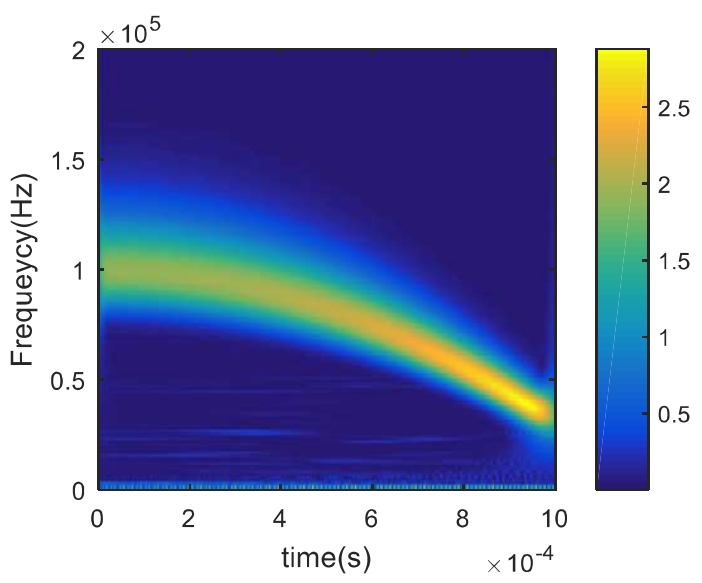

(a)

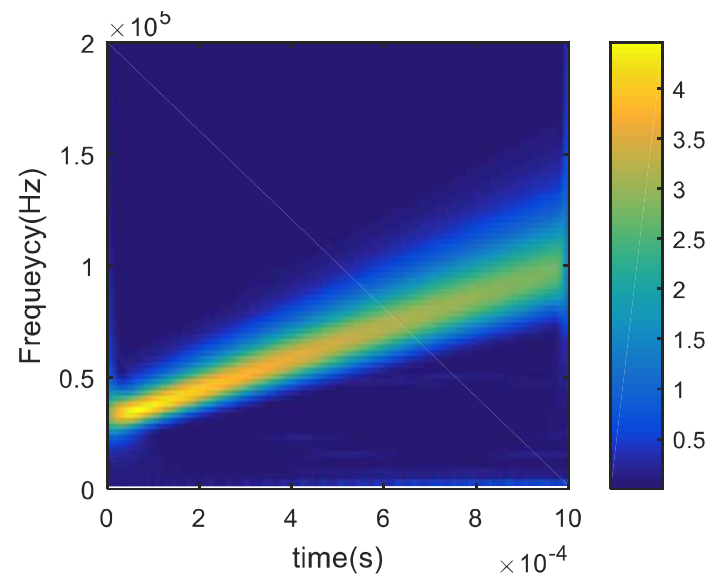

(b)

Figure 8. Chirp signals in the range of $30 \mathrm{kHz}-100 \mathrm{kHz}$, (a) convex mode, (b) linear mode.

To validate the numerical model, pipe geometry and materials similar to an example shown in [31] are modeled. To prevent the reflection from the boundaries, the excitation signal is placed at the middle of the pipe, and the strain energy density at a particular time is plotted in Figure 9. Both models have similar wave envelope and dispersion at a particular point on the pipe.

[Graphic image withheld. Please contact the author]

Figure 9. Assessment of numerical model with [31]. 
The wave type studied are the guided elastic waves, which are guided by the finite dimensions of the test object and can travel long distances with lower energy loss than other wave modes. In particular, wave propagation characteristics of three wave types in a typical pipe found in nuclear power plants are reported: torsional waves, SH waves, and non-axially symmetric waves.

\subsubsection{Torsional Waves}

First, we studied the torsional waves. For long-range propagation on piping, these waves are often preferred because they exhibit less dispersion than the other wave modes. The comb-type transducer is considered in the model. To generate pure torsional wave, an array of annular comb transducers is placed around the pipe circumference with the effective element width as $1.4 \mathrm{~mm}$, and the space between each element as $1.4 \mathrm{~mm}$. Circumferential displacement responses of five typical locations are investigated to show the wave propagation along the pipeline (Figure 10). As the purpose of this study is to understand the mechanism of wave propagation, the frequency domain analysis is adequate to show the decay of signal energy. The response signals under both quadratic convex and linear chirp are shown in Figure 11. The excitation signals and response signals are compared in the same figure. According to the response signal, the excited frequency signals can be preserved in the frequency domain, especially for low frequency components. With the increase of frequency, the response signals dissipate faster. This phenomenon can be explained by the dispersion curve (Figure 5). For reducing the decay of higher frequency component, the chirp signal in convex mode has been identified as the better option.

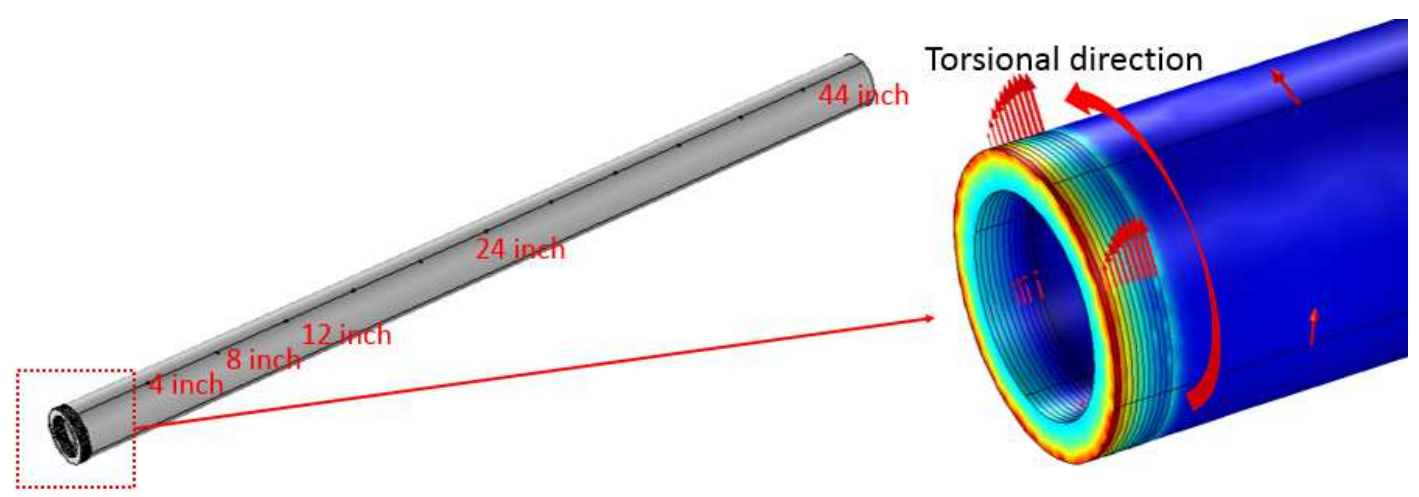

Figure 10. Pipe model and excitation of torsional wave mode. 


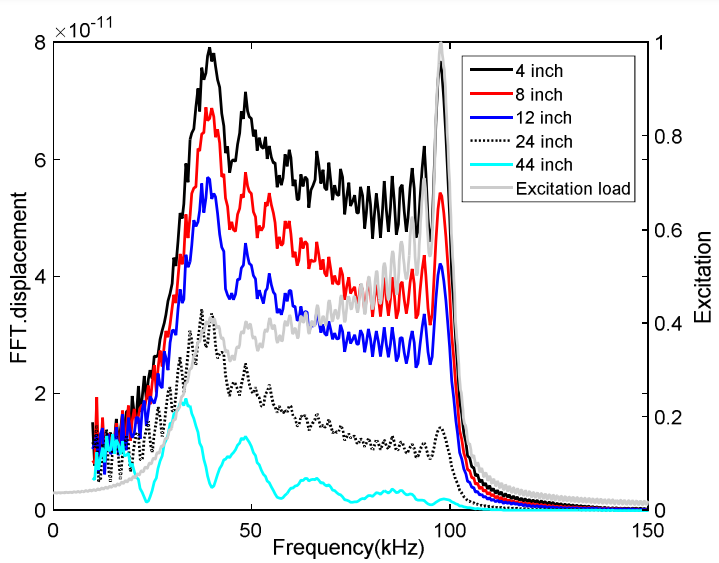

(a)

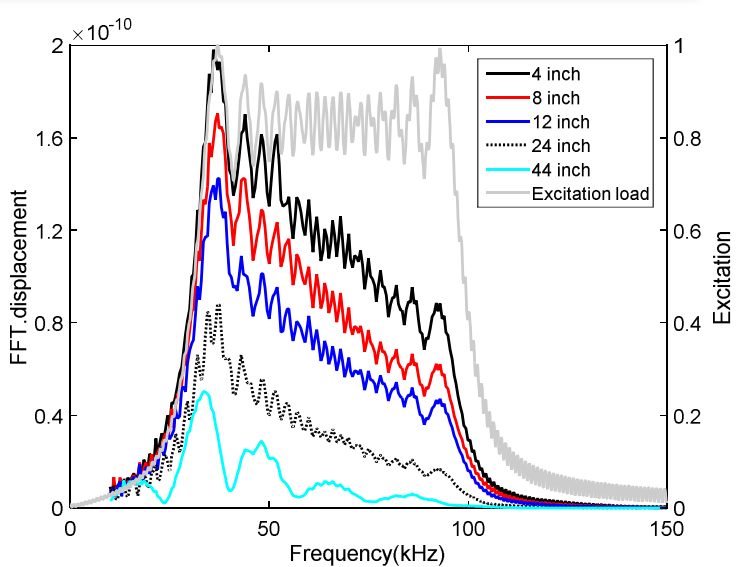

(b)

Figure 11. Responses of circumferential displacement at different locations, (a) under convex mode and (b) under linear mode.

\subsubsection{SH Waves}

Shear horizontal $(\mathrm{SH})$ waves are generated by the particle motion in the in-plane direction, while the wave propagation is orthogonal to the particle motion. SH wave mode can be excited by the EMATs. Usually EMAT has two major parts: a magnet and a mender-line coil, which can generate a strong horizontal Lorentz force to cause the particle motion in the tested object. EMAT transducer generates a particular frequency by tuning the length of $\lambda$, which is the wavelength of the desired frequency, as shown in Figure 12a. The EMAT design in this figure can be used to excite the frequency of $250 \mathrm{kHz}$ of the fundamental mode $\left(\mathrm{SH}_{0}\right)$ [38]. In this model, the Lorentz force is simplified as three pairs of line load, as shown in Figure 12b. Two models are used to show two directions of $\mathrm{SH}$ wave propagation: one is along the axial direction, and the other is circumferential direction. Independent from the direction, the response displacement is the same as particle motion. For analyzing the $\mathrm{SH}$ wave along axial direction, the model is the same as that used in connection with Figure 9. The only difference is the method of loading. The source function is the linear chirp shown in Figure 7b. The response signals are shown in Figure 13. The response signals show similar trend as torsional wave. The lower frequency component dissipates less than higher frequencies. However, the response around $78 \mathrm{kHz}$ disappears because the simulated EMAT is tuned as excitation of $92 \mathrm{kHz}$, which coincides with the second peak frequency. The difference can also be from the error due to simplification of EMAT sensor in the model.

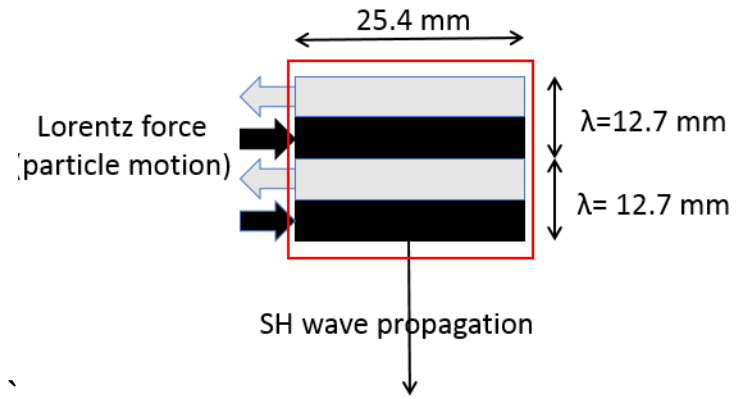

(a)

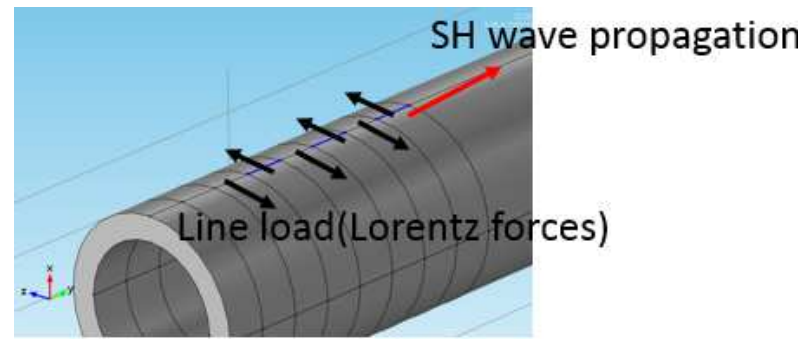

(b)

Figure 12. (a) EMATs design, (b) EMATs simplified model. 


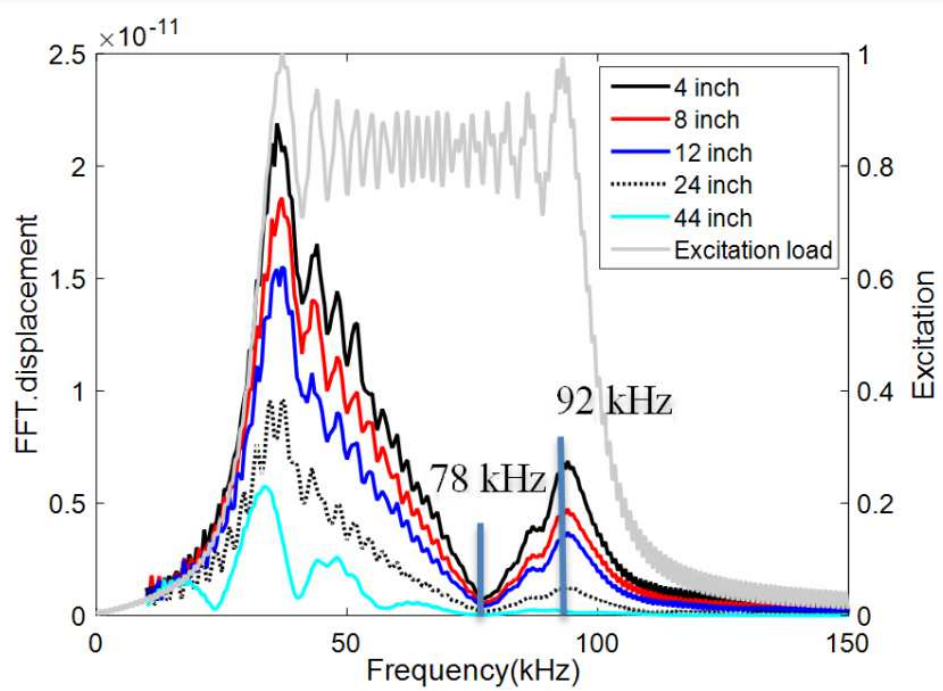

Figure 13. Response signals due to axial SH wave mode.

To understand the SH wave propagation behavior better, a larger model to show $\mathrm{SH}$ wave propagating in the circumferential direction is also presented. The dimension of the model is from [31], which is shown in Figure 14. In this model, frequency of EMAT is tuned as $93 \mathrm{kHz}$, and also the tone burst signal with $100 \mathrm{kHz}$ is applied. The comparison of excitation signal and response is shown in Figure 15. The frequency component of excitation can be preserved, while only a small shift toward the lower frequency is observed. Therefore, after proper tuning, the $\mathrm{SH}$ wave can also preserve its frequency and travel over long range.
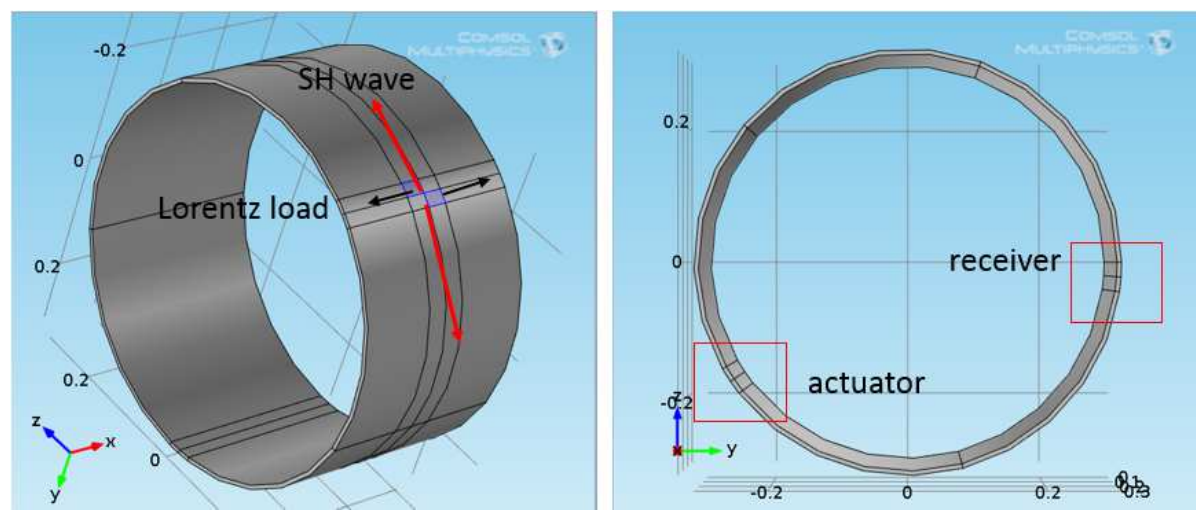

$\mathrm{DO}=0.6096 \mathrm{~m}$ $\mathrm{TW}=0.00635 \mathrm{~m}$ Length $=0.3048 \mathrm{~m}$

Figure 14. Circumferential SH wave propagation modeling. 


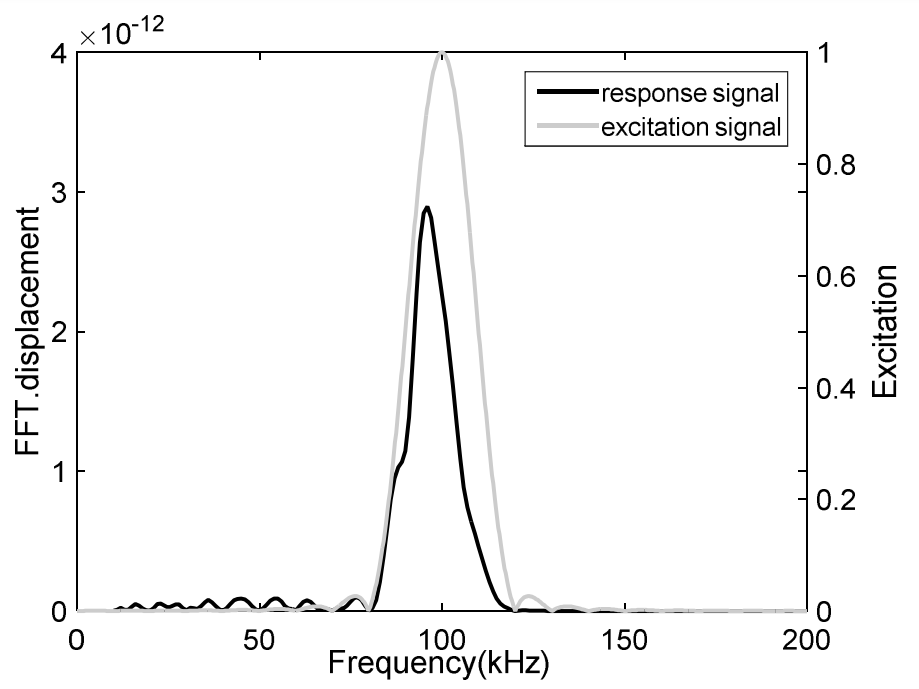

Figure 15. Response signal due to circumferential SH wave mode.

\subsubsection{Non-axisymmetric Waves}

Non-axisymmetric waves are excited by a direct transducer contact on a finite area on the pipe, which is the superposition of axisymmetric longitudinal modes $L(0, n)$ and non-axisymmetric modes $F(m, n)$ with different amplitudes and phase velocities [34]. In the numerical model, the point load excitation is adopted. The model is the same as that used in connection with Figure 9. The linear chirp is the loading function, which is the same as that shown in Figure 7b. The point load could trigger more complex waves including longitudinal, torsional, and flexural modes. The flexural wave is salient due to non-axisymmetric motion of particles. The response signals in flexural mode are distorted after they travel a short distance (Figure 16). Unlike waves in torsional and $\mathrm{SH}$ modes, the non-axisymmetric waves in flexural mode cannot preserve the source information. Instead, the response signals show a more complex behavior.

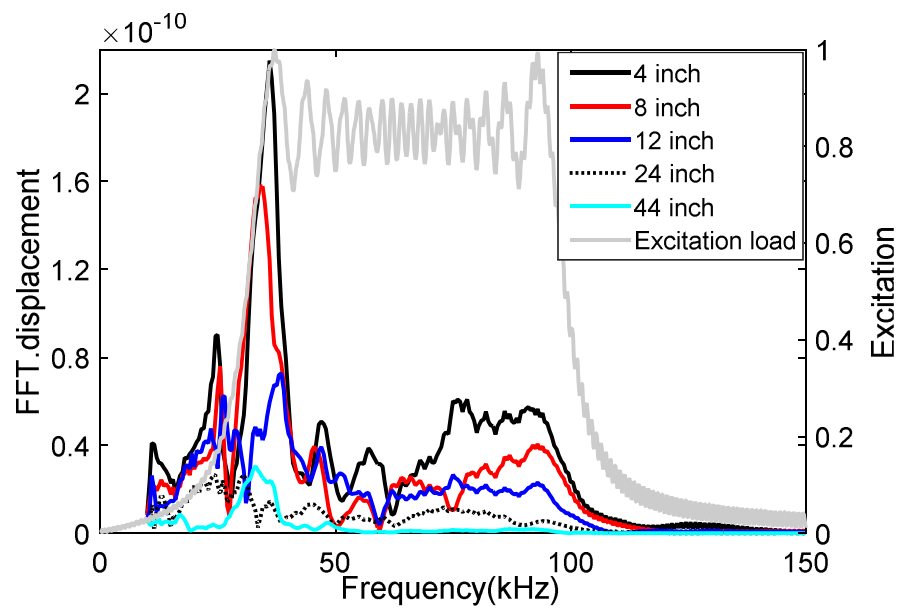

Figure 16. Response signal in non-axisymmetric wave mode.

\subsubsection{Influence of Elbow on Ultrasonic Wave Propagation}

Mode conversion occurs when an ultrasonic waves passes through a bended pipe [39]. To understand the influence of elbow on the torsional wave propagation, the pipe model with 90 degrees elbow is modeled (Figure 17a). The material properties and dimension of the pipe are still 
the same as those listed in Table 2. The Hanning windowed tone burst signal with the frequency of $30 \mathrm{kHz}$ is introduced as the loading function. In this case, time domain analysis is used to better describe wave propagation in the pipeline with elbow. Figure $17 \mathrm{~b}$ shows the torsional wave propagation through the elbow. After interacting with the elbow, the wave front is distorted due to the reflection and transmission by the elbow; and mode conversions occur simultaneously. The loading function together with the response signals of receiver A, B and C are shown in Figure 18. The peak frequency of the response signal shifts away from $30 \mathrm{kHz}$ to form another two frequency modes. The propagating waveform interferes with the reflected waveform. Therefore, a window was defined to reduce the influence of backward reflection of the waveform. The defined window is marked in Figure 18a. The elbow influence is illustrated more clearly in Figure 19. As shown in Figure 19b, the response signal of receiver A has the same peak frequency as excitation load, while the peak frequencies of receiver $\mathrm{B}$ and $\mathrm{C}$ are shifted. This phenomenon shows that wave mode conversion happens after the wave travels through the elbow, which has been verified in [39].

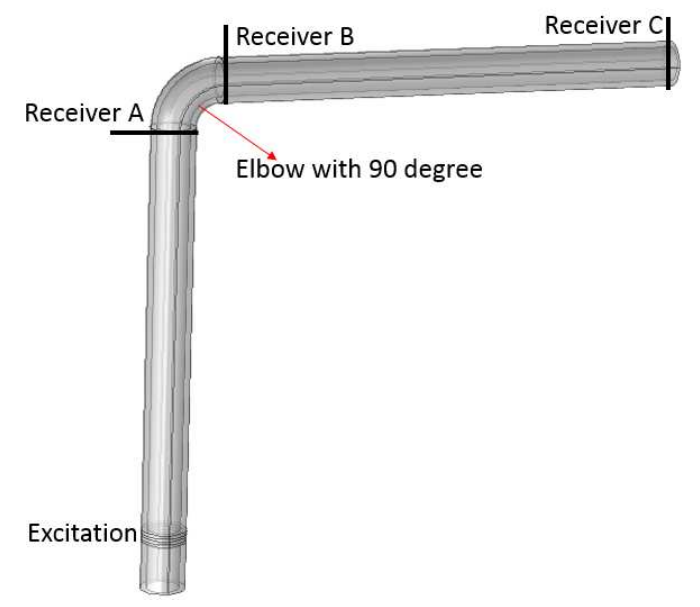

(a)

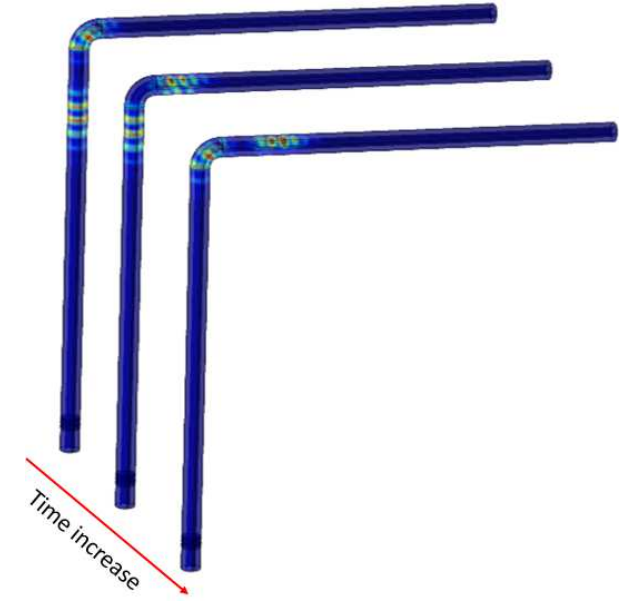

(b)

Figure 17. (a) The model of pipeline with elbow, (b) wave group propagation near the elbow.

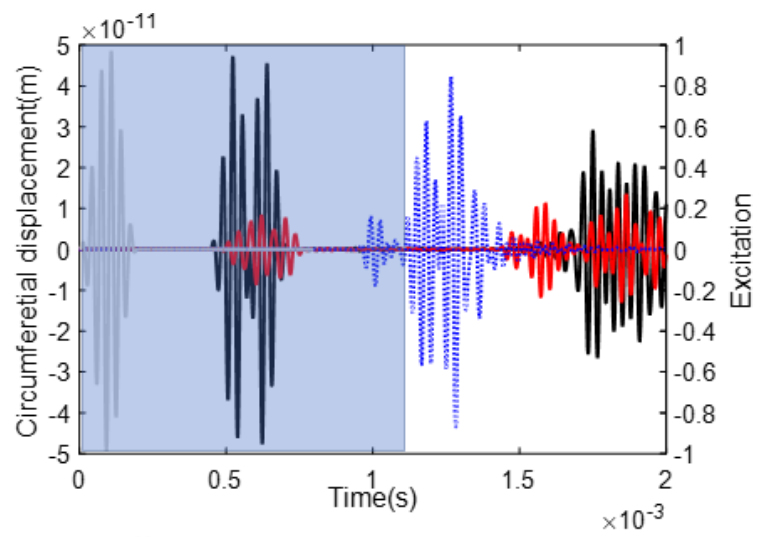

(a)

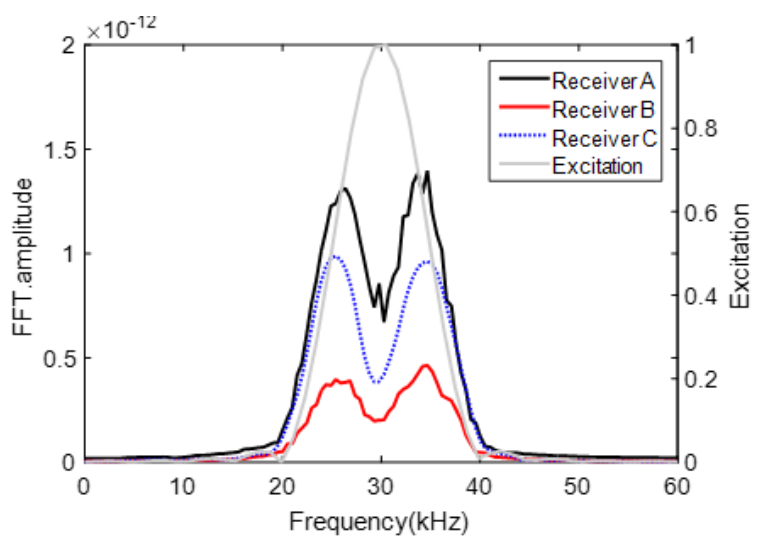

(b)

Figure 18. Response signals of the pipeline model with elbow, (a) time domain (b) frequency domain. 


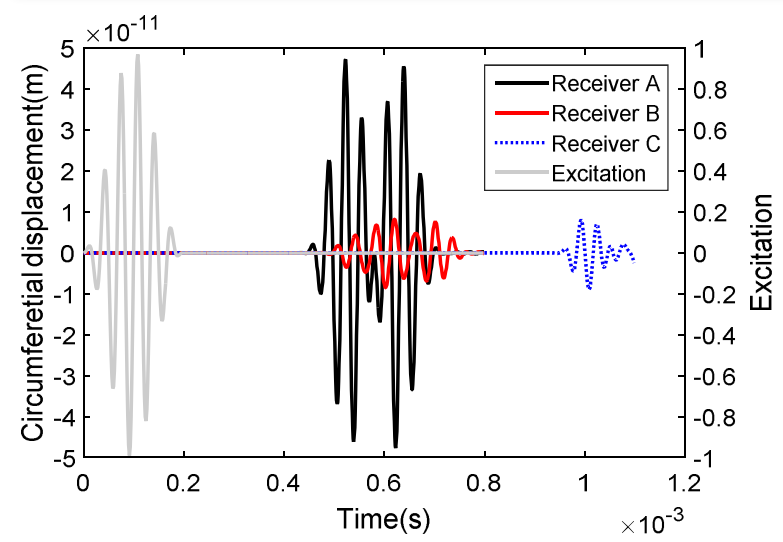

(a)

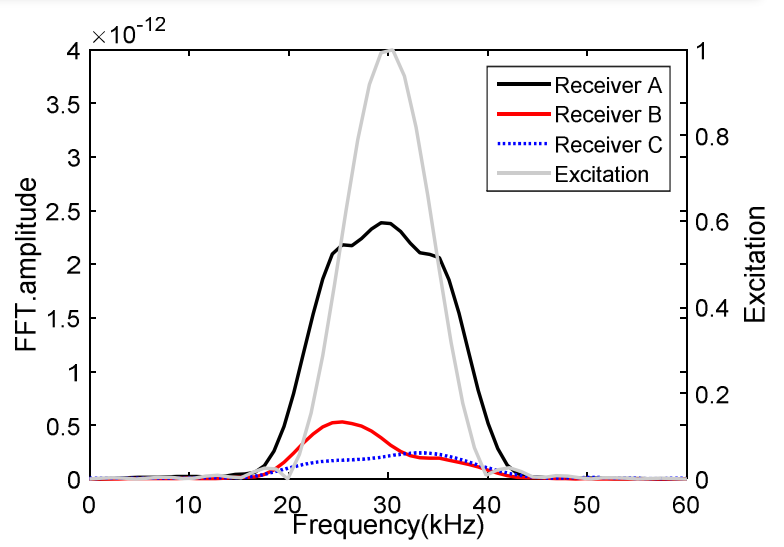

(b)

Figure 19. Windowed response signals, (a) time domain (b) frequency domain.

\subsection{Discussion}

Though any wave mode can be used for transmitting waves in pipes by comprehensive tuning, it is difficult to identify and restore the excitation signals based on the non-axisymmetric waves in flexural modes. Therefore, axisymmetric modes are preferable because their acoustic distribution in the structure is relatively simple. For mutual communication of wave information, dispersion free wave modes (torsional and SH waves) allow the wave to propagate over longest distances with minimum distortion.

\section{ULTRASONIC COMMUNICATION SYSTEM}

\subsection{Experimental setup}

Initial experiment efforts have focused on identification of appropriate transducers and coupling mechanism for transmission and reception of acoustic signals. In particular, the performance bounds on information transmission along a pipe using a pair of commercially available PZTs and EMATs were evaluated.

In Figure 20, the main components of the current experimental acoustic system setup, i.e. an arbitrary waveform generator (AWG) and power amplifier (PA) on the transmission side, and a low-noise amplifier (LNA), a filter and data acquisition system on the receiver side, are schematically represented. The laboratory apparatus showing various components of the acoustic communication system is displayed in Figure 21. The PZTs are coupled to the test piece using angle wedges, which allow adjustment of the beam angle. 


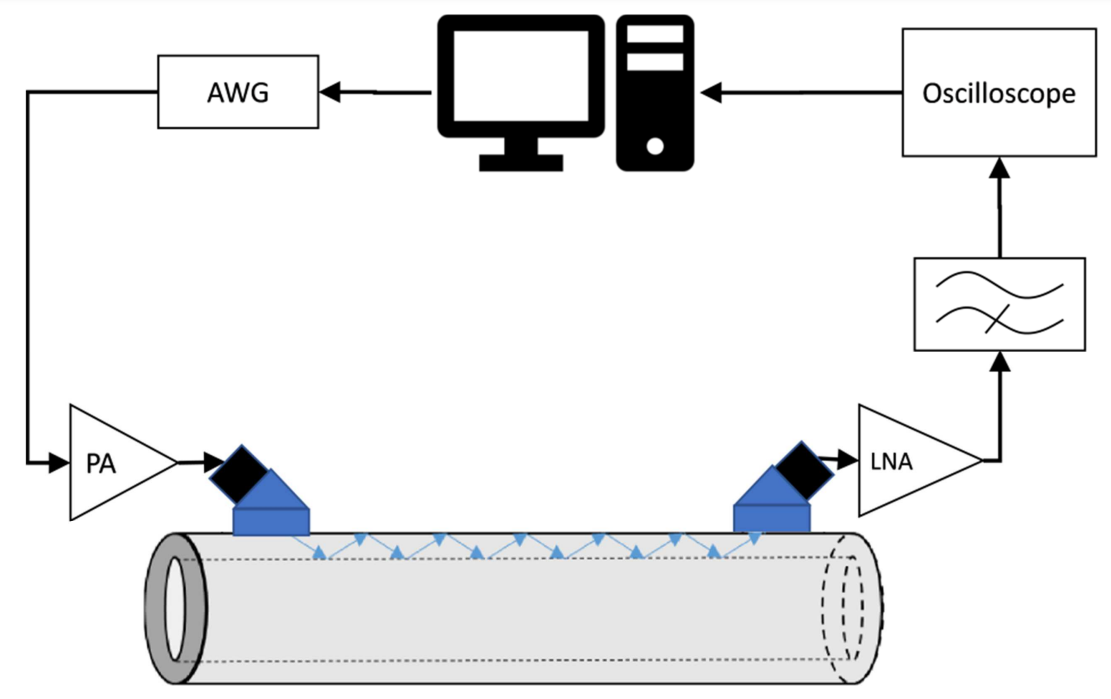

Figure 20. Schematic of the laboratory system for evaluation of acoustic communication along pipes and other metal pathways.

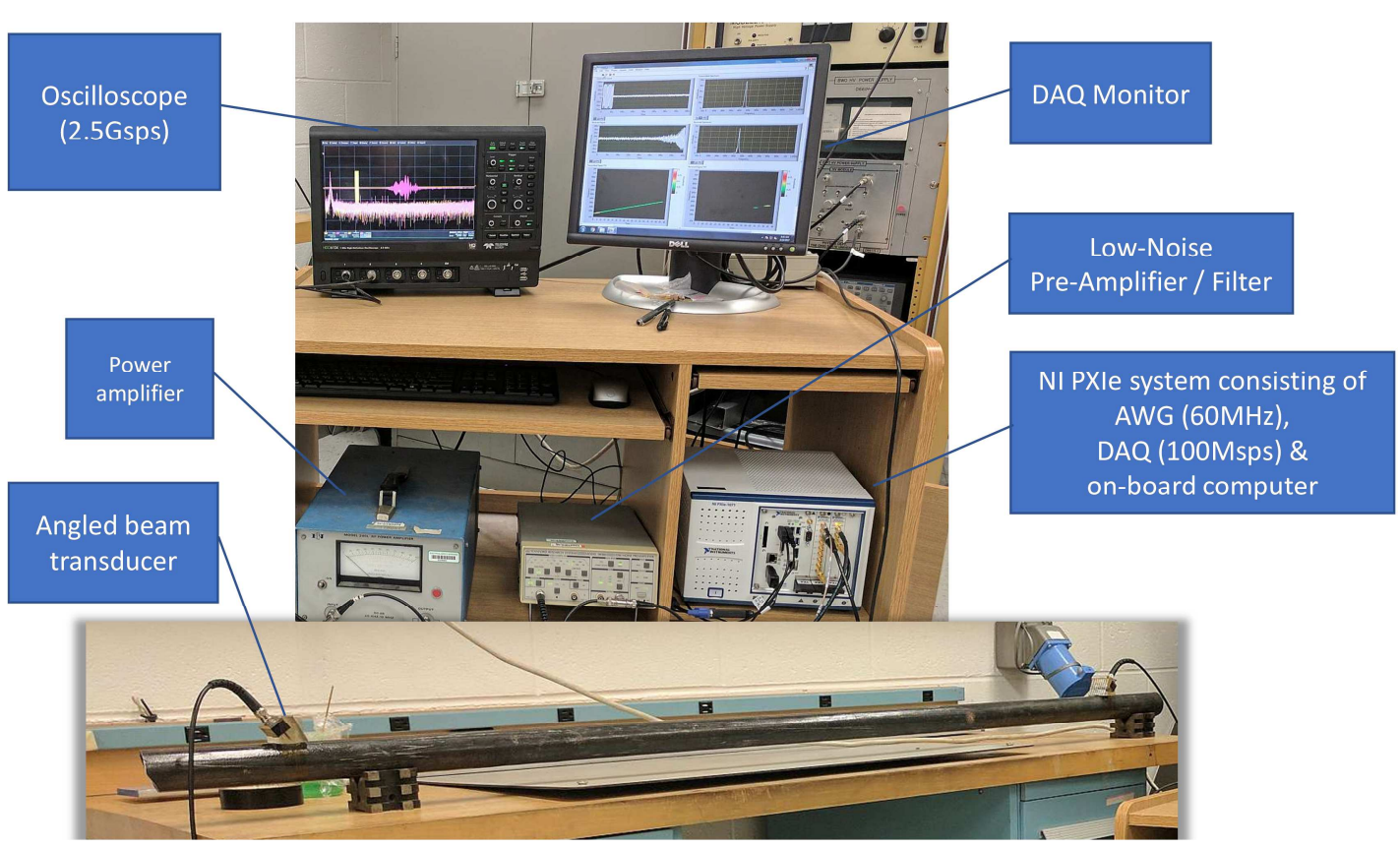

Figure 21. Photo of the laboratory setup showing various components of the acoustic communication system. Displayed at the bottom is the pipe mounted with angled transducers.

\subsubsection{Pipe}

For the initial experimental studies, a five-foot long schedule-160 stainless steel pipe with 2.375" outer diameter and 0.344 " wall thickness was chosen as the medium for evaluation of acoustic signal transmission. As for the length of the adopted pipe (5 feet), it represents the minimum distance required for transmission of signals along a CVCS pipe, which penetrate through reactor containment wall. 


\subsubsection{Transducers}

Olympus paintbrush PZTs with resonant crystal frequency of $500 \mathrm{kHz}$ and approximately $\pm 10 \%$ $3 \mathrm{~dB}$ bandwidth mounted on angled wedges were chosen for initial testing. The lowest frequency for commercially available transducers of this type is $500 \mathrm{kHz}$. As discussed in the previous section, a lower frequency results in lower signal attenuation and allows for more power-efficient operation of the communication system. The transducers provide a relatively linear frequency response in the $400 \mathrm{kHz}$ to $600 \mathrm{kHz}$ range when driven with linearly swept chirp pulses.

The PZT angled beam transducers use the principles of refraction and mode conversion to produce refracted shear or longitudinal waves in the test material. The incident angle necessary to produce a desired refracted wave can be calculated from acoustic's Snell's Law

$$
\frac{\sin \theta_{\mathrm{i}}}{C_{i}}=\frac{\sin \theta_{r l}}{C_{r l}}=\frac{\sin \theta_{r s}}{C_{r s}}
$$

where

$\theta_{\mathrm{i}}:$ Incident Angle of the Wedge

$\theta_{\mathrm{rl}}:$ Angle of the Refracted Longitudinal Wave

$\theta_{\mathrm{rs}}$ : Angle of the Refracted Shear Wave

$\mathrm{C}_{\mathrm{i}}$ : velocity of the incident Material (Longitudinal Wave)

$\mathrm{C}_{\mathrm{rl}}$ : material sound velocity (longitudinal)

$\mathrm{C}_{\mathrm{rs}}$ : velocity of the test material (Shear)

In Figure 22, acoustic wave refraction of a longitudinal wave excited by an angle beam transducer is shown. In Figure 23, the diagrams of relative intensities of longitudinal and shear waves in stainless steel generated via refraction from an acrylic wedge are displayed. For the tests conducted in this project, the angle of the wedge is $33^{\circ}$, which results in the highest degree of coupling into refracted shear wave in the pipe used in these tests.

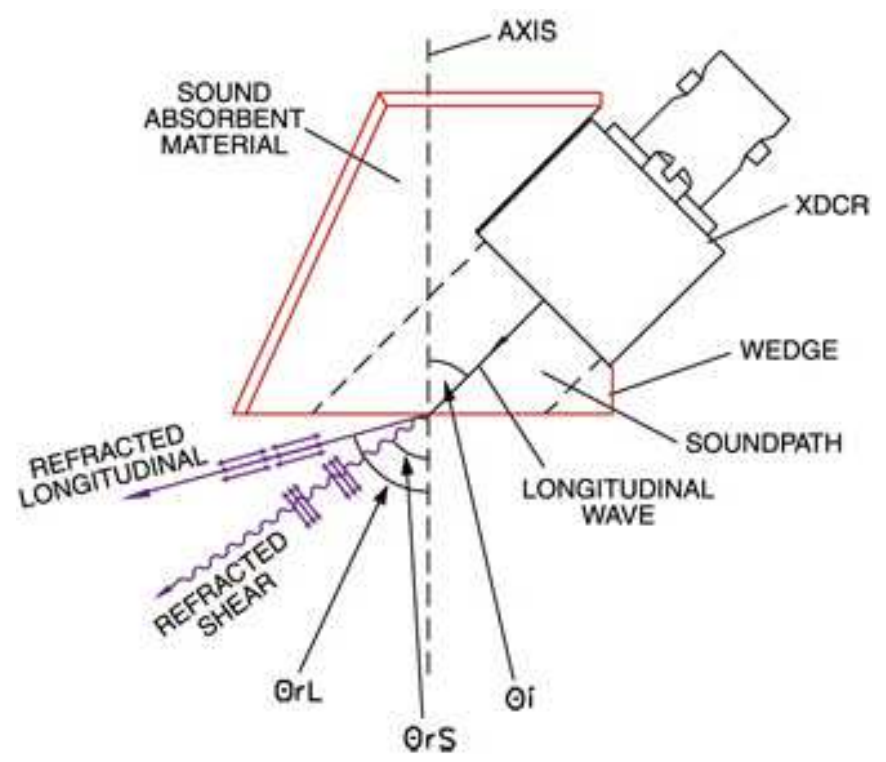

Figure 22. Snell's Law of angle beam transducer. 


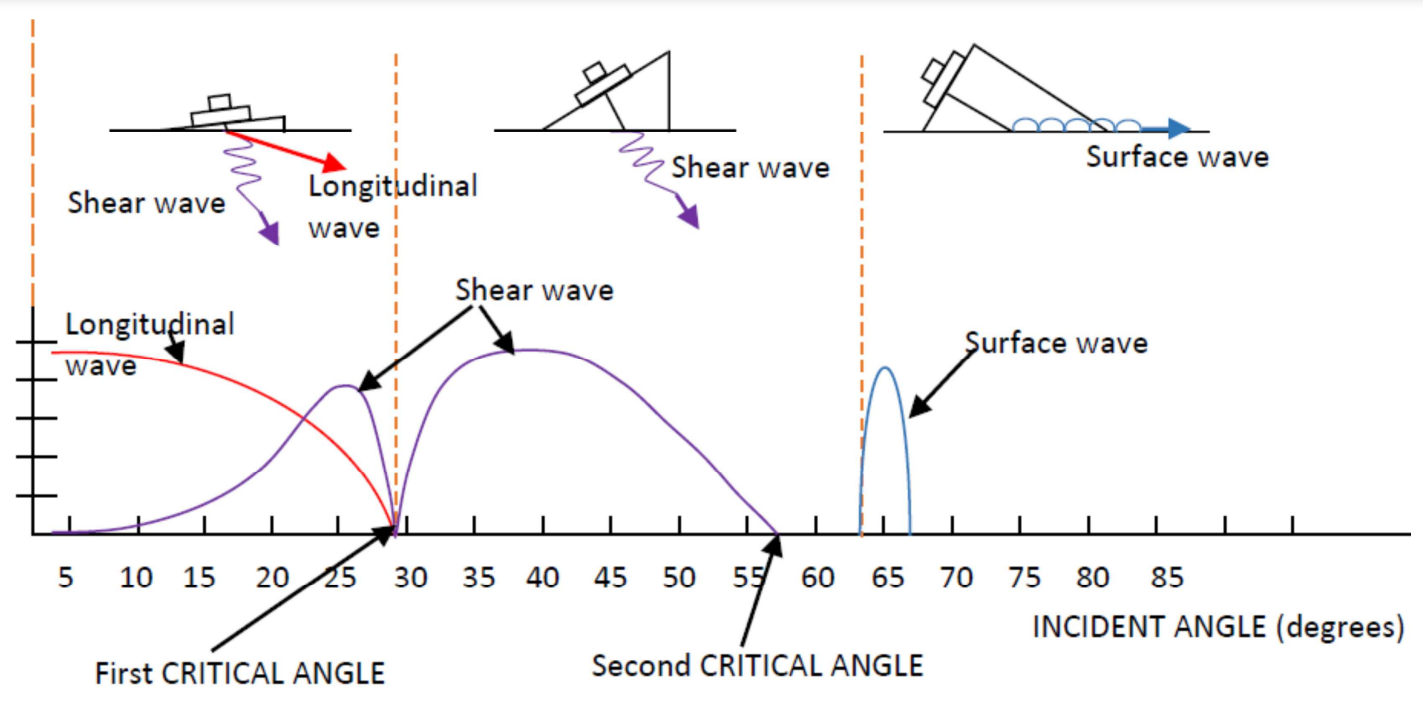

Figure 23. Relative amplitude of wave modes.

\subsubsection{Electronics}

The AWG is used to generate pulses of around $0.1 \mathrm{~ms}$ duration composed of sinusoidal waves at different frequencies. The signal is then amplified by the PA and fed into the transducer. After propagating through the medium - stainless steel pipe or plate used in this study - the signal is received at the other end by a matched identical transducer. The received signal is subsequently amplified and acquired by an analog to digital convertor (ADC).

\subsubsection{Data acquisition}

All data acquisition and analysis are currently performed under LabView ${ }^{\mathrm{TM}}$ software. A virtual instrument has been developed for this purpose which handles all time and frequency domain analysis of the data (Figure 24). The spectrum is obtained by accumulating the data from each one of the selected frequencies, representing the frequency response of the transmission medium. The interface also displays in real time the transient evolution of the spectrum, in part to help identify unwanted resonant modes excited by the transducers. 


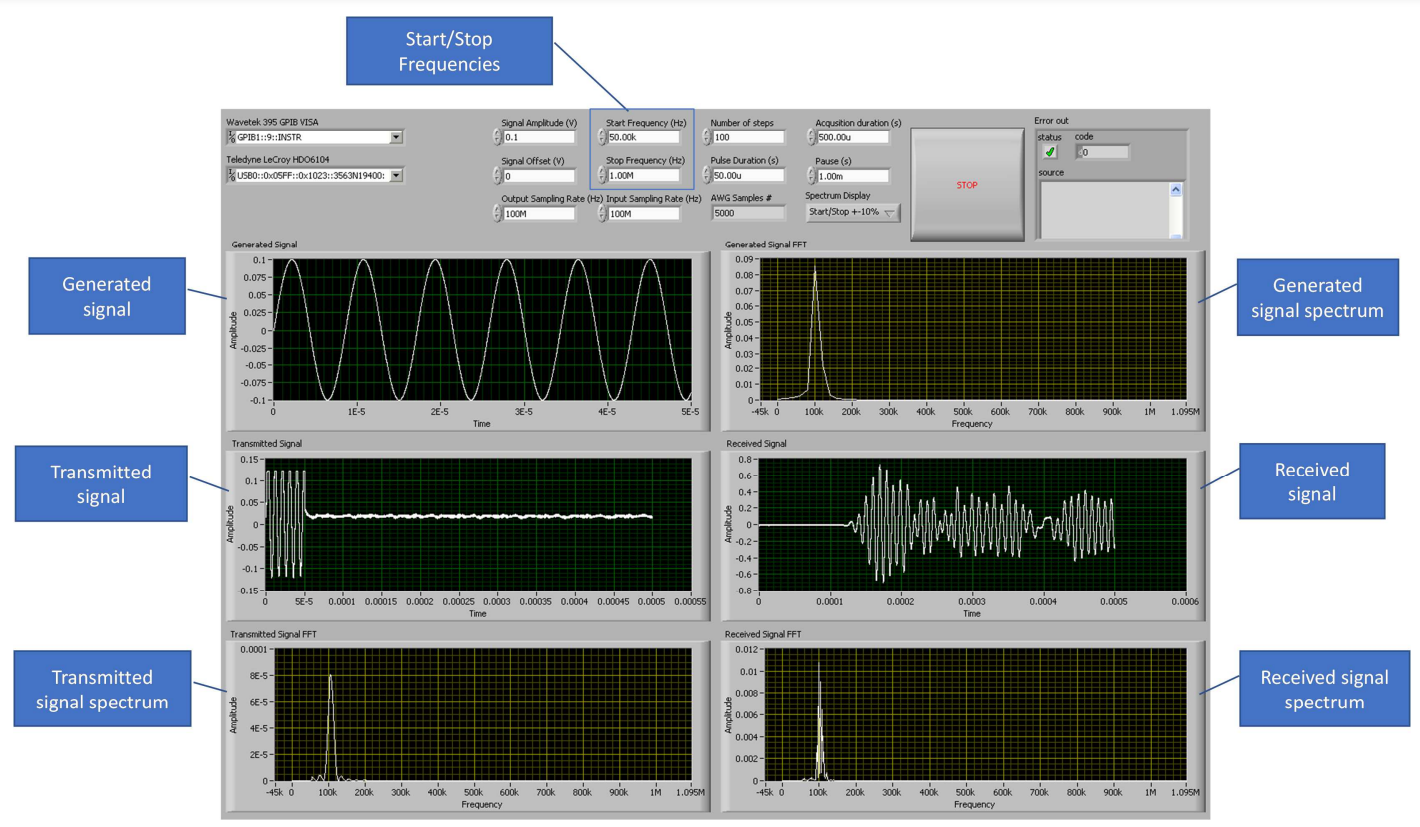

Figure 24. Virtual instrument developed under LabView ${ }^{\mathrm{TM}}$ to generate, detect and analyze acoustic/ultrasonic signals.

\subsection{Evaluation of modulation methods in PZT-based experimental setup}

Preliminary evaluations have been conducted of frequency modulation (FM) and on-off keying (OOK) modulation. The objective is to determine optimal modulation scheme for transmission of signal under the constraint of limited signal generator power.

\subsubsection{Frequency distortion}

Feasibility of frequency modulation (FM) scheme for information transmission was evaluated using spectral dispersion characteristics of pulses of different temporal duration. Figure 25 demonstrates that transmitting a narrowband $500 \mathrm{kHz}$ signal with $50 \mu$ s duration results in more spectral dispersion, as compared to the case when the pulse duration is $100 \mu \mathrm{s}$. Given the amount of spectral distortion and dispersion observed in preliminary tests, we conclude that FM not likely going to be the optimal coding scheme in this project.

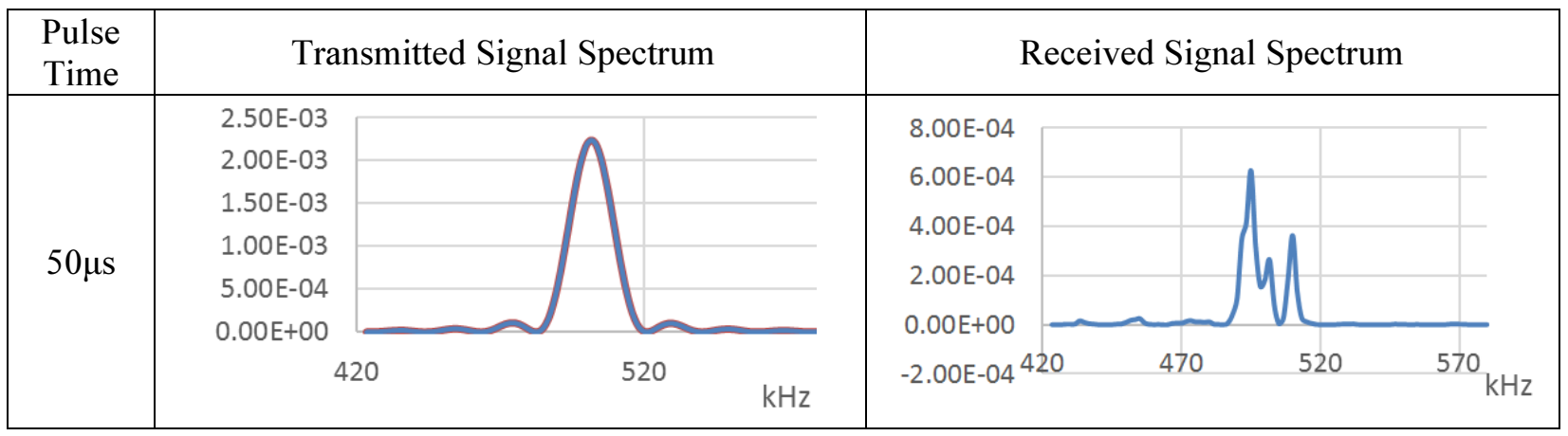




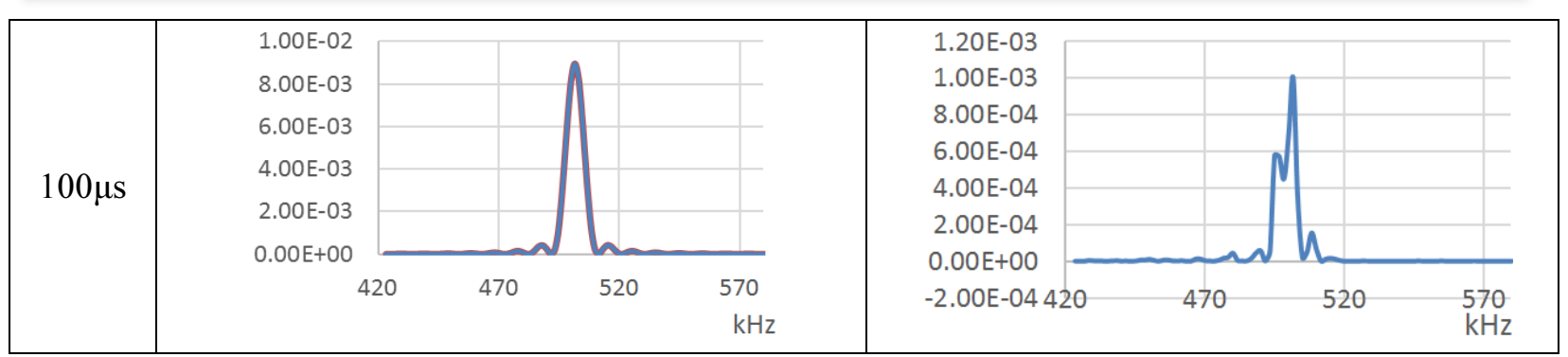

Figure 25. Spectral dispersion and distortion of narrow band pulses characterized by different temporal durations.

\subsubsection{OOK modulation}

On-off keying (OOK) is a common scheme for time-domain encoding of information in communication systems with frequency narrowband channels. Preliminary evaluation of OOK channel characteristics by transmitting variable duration pulses were conducted. Shorter pulse duration results in higher data transmission bit rate (BR). However, smaller pulse energy may result in poor signal recovery leading to high bit error rate (BER). In Figure 26, the results of transmission and reception of a train of $50 \mu$ s duration OOK pulses are displayed. In Figure 27, the results of transmission and reception of a train of $100 \mu$ s duration OOK pulses are displayed. All system settings, such as LNA gain, were kept the same throughout the study. Based on the preliminary results, we conclude that OOK scheme with low BER can be achieved using $50 \mu$ s duration pulses. This is equivalent to $20 \mathrm{kbs}$ data transmission rate.

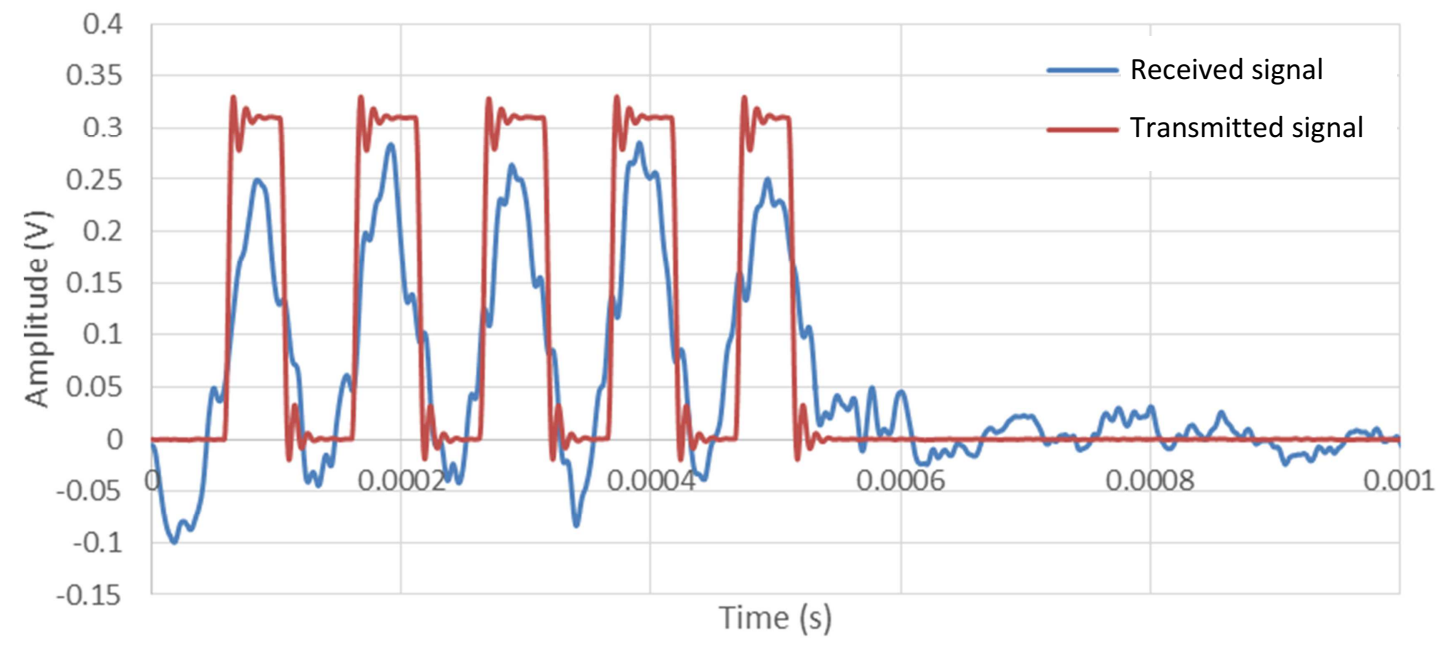

Figure 26. Transmission and reception of a train of $50 \mu$ s duration $00 \mathrm{~K}$ pulses. 


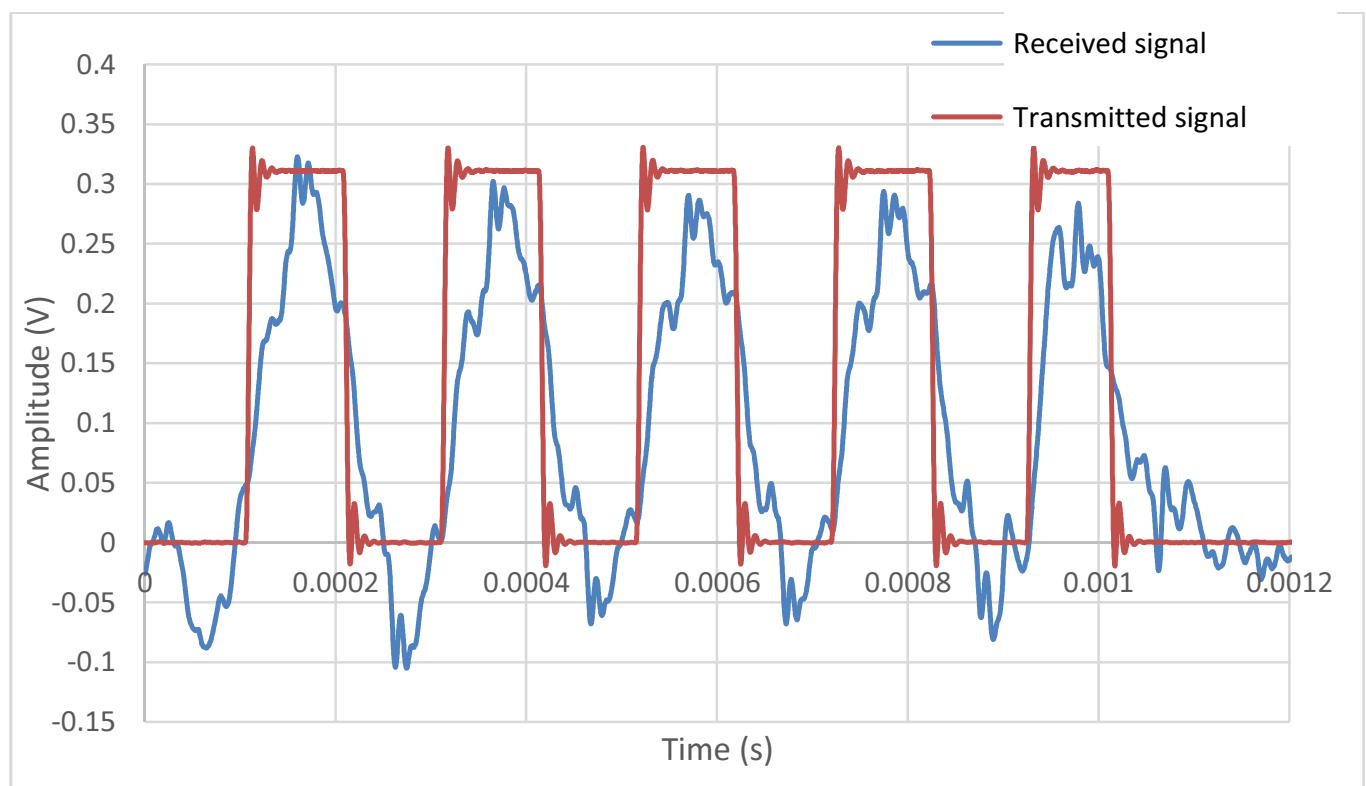

Figure 27. Transmission and reception of a train of $100 \mu$ s duration $00 \mathrm{~K}$ pulses.

\subsubsection{ASK modulation}

Amplitude shift keying (ASK) is a common implementation of OOK communication protocol. The principle of ASK is to code signal $s(t)$ as

$$
s(t)= \begin{cases}1 & \operatorname{logic} 1 \\ 0 & \operatorname{logic} 0\end{cases}
$$

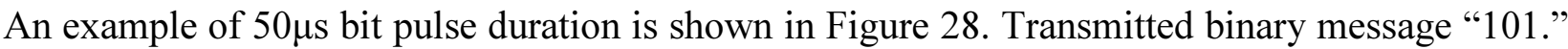
Transmitted and received signals are overlaid by subtracting time-of-flight from received signal. Amplitudes of transmitted and received signals are not to scale.

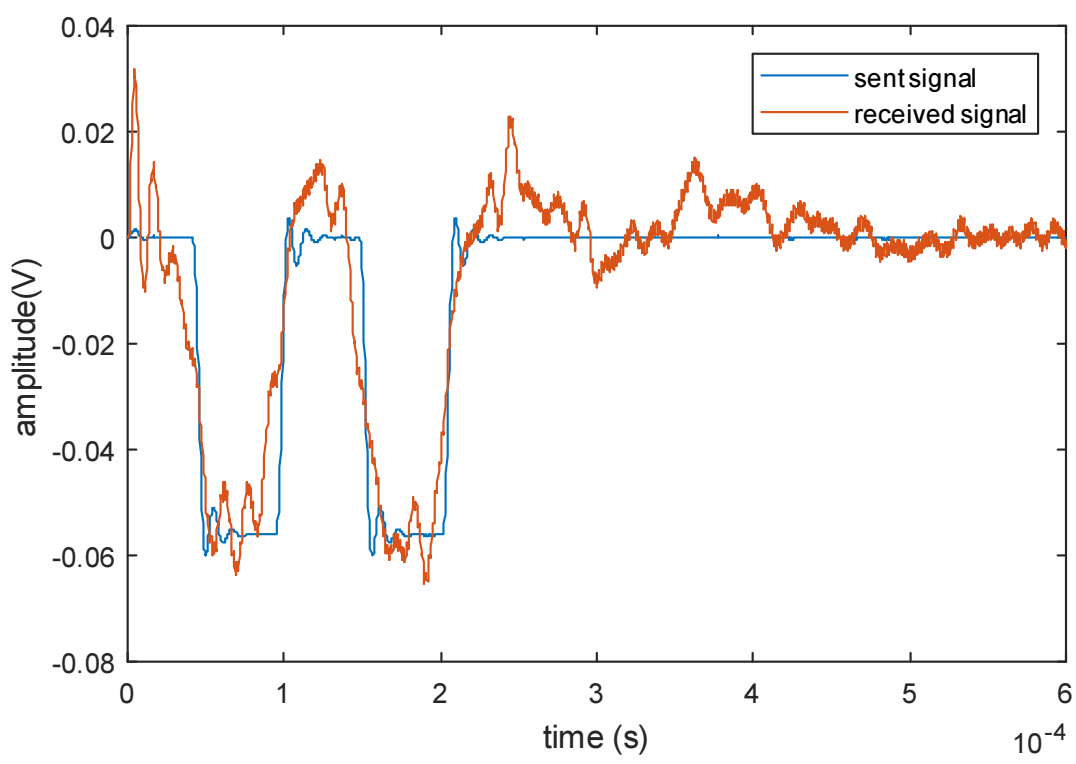

Figure 28. Example of ASK modulation with $50 \mu$ bit pulse duration. Transmitted and received signals are overlaid with amplitudes not to scale. 
More complicated ASK waveforms are shown in Figure 29. In the top panel, bit pulse length is $50 \mu \mathrm{s}$. The transmitted binary message is "11001011." In the bottom panel, the binary signal is "1101011".

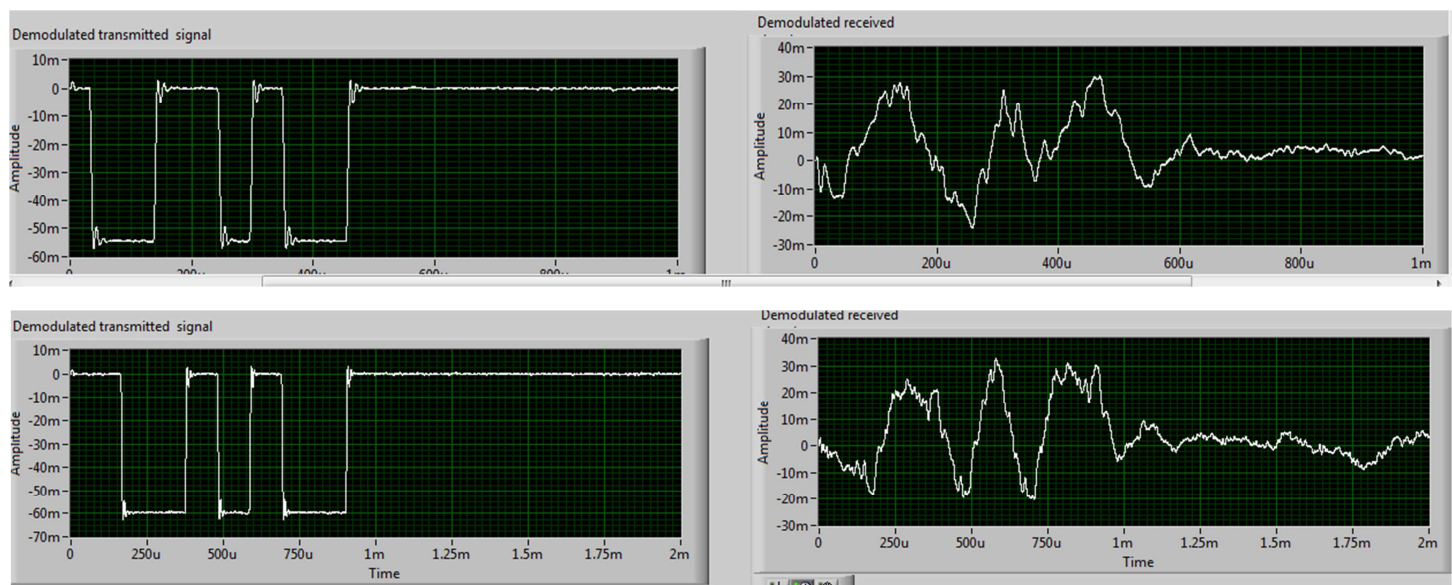

Figure 29. Examples of ASK transmitted signals (on the left) and received signals (on the right). Top panel: binary signal "11001011". Bottom panel: binary signal "1101011".

\subsubsection{PSK modulation}

The modulation method of the phase shift keying (PSK) is almost the same as that of the ASK. The difference is that the $\mathrm{s}(\mathrm{t})$ signal is the imaginary part of IQ signal, and ' 1 ' represent 'logic 1'; '-1' represent 'logic 0'. Figure 30 gives an example of PSK signal modulation with bit pulse duration of $50 \mu \mathrm{s}$. Transmitted binary signal is " 101 ". Additional examples of more complex PSK coded sequences are shown in Figure 31.

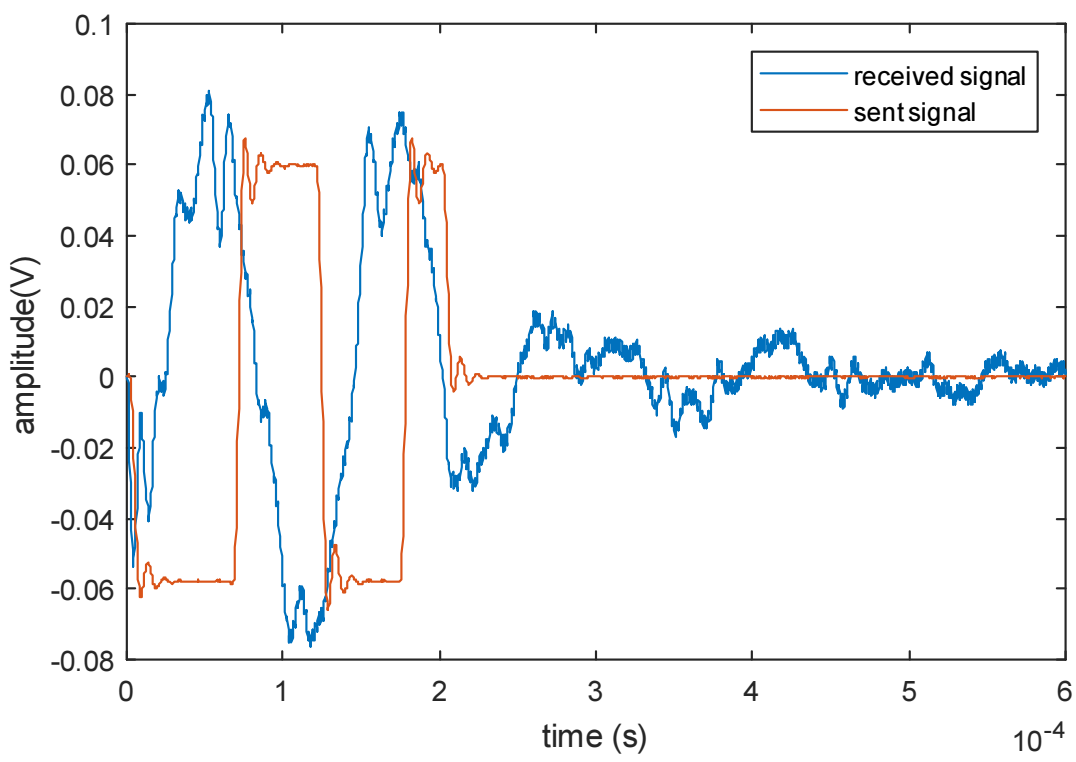

Figure 30. Example of PSK signal with 50 micro-s bit pulse duration. 


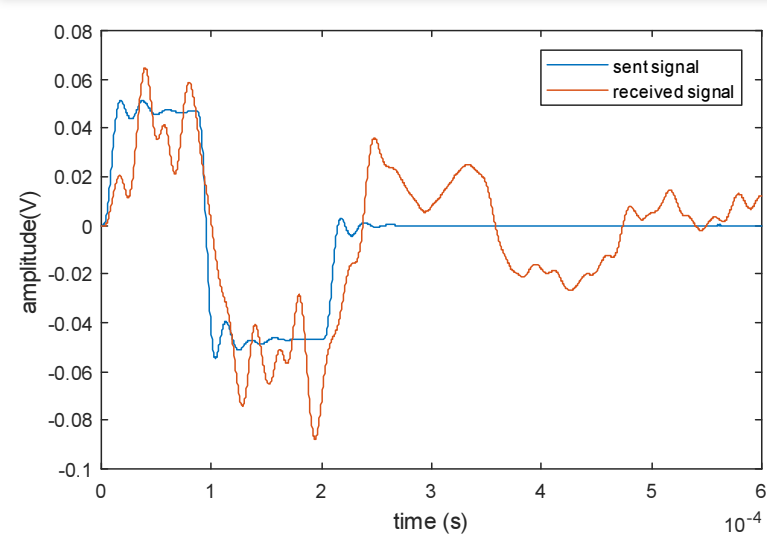

(a)

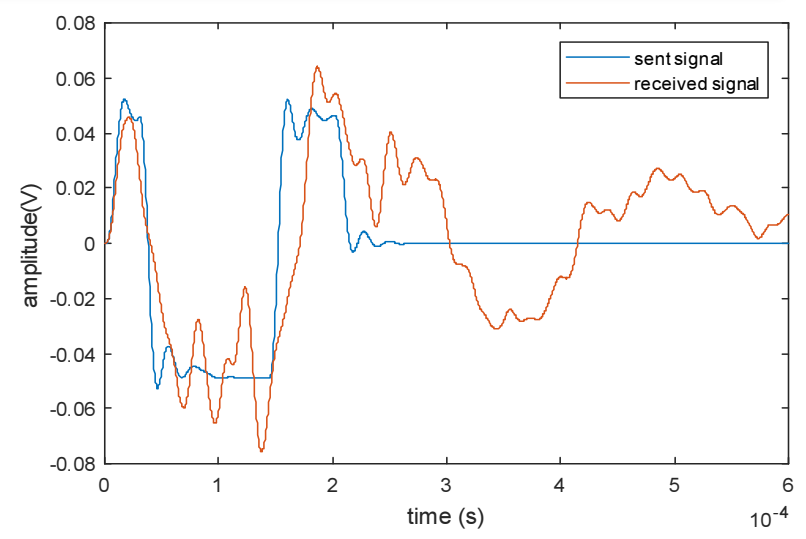

(b)

Figure 31. Example of PSK modulation, (a) binary signal "1100", (b) binary signal "1001".

\subsection{Signal transmission with EMAT}

As described in previous report, EMAT transducers generate ultrasonic waves in electrically conductive materials by Lorentz force, by electro/magnetostrictive effect, or by a combination thereof. Acoustic waves are generated directly within the electrically conducting medium instead of inside the transducer as with piezoelectric transducers. The Lorentz force $\left(\overline{\mathrm{F}}_{\mathrm{L}}\right)$ is caused by interaction between the current density $(\bar{J})$, which is inducted through the eddy current coil, and the magnetic flux $\left(\bar{B}_{0}\right)$. The direction and intensity of the force is determined by the vector equation:

$$
\overline{\mathrm{F}}_{\mathrm{L}}=\overline{\mathrm{J}} \times \overline{\mathrm{B}}_{0}
$$

The incident angle of SH-EMAT is determined as

$$
\sin (\alpha)=\lambda / \lambda_{s}
$$

where $\lambda$ is the wavelength of the SH- wave, and $\lambda_{s}$ is the unit dimension of the magnet. For the initial tests, an Innerspec EMAT generating SH waves was used as transmitter and a PZT was used as receiver. Because of the RF coupling associated with EMAT excitation, a time delay was introduced to avoid interference of the RF pulse with the test signal. The optimum angle of the PZT receiver was obtained by using a variable angle wedge. Furthermore, the optimum carrier frequency was selected based on maximization of the received signal amplitude transmitted along the pipe. In Figure 32, the spectrum of transmitted single pulse signal from EMAT to PZT is shown. In Figure 33, the time-domain waveform of a signal transmitted from EMAT to PZT is shown. 




(a)

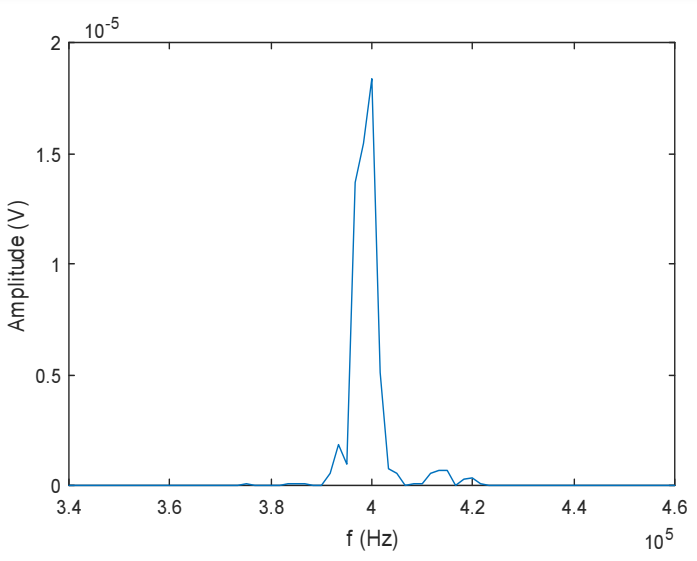

(b)

Figure 32. (a) EMAT transmitted signal spectrum, (b) PZT received signal spectrum.
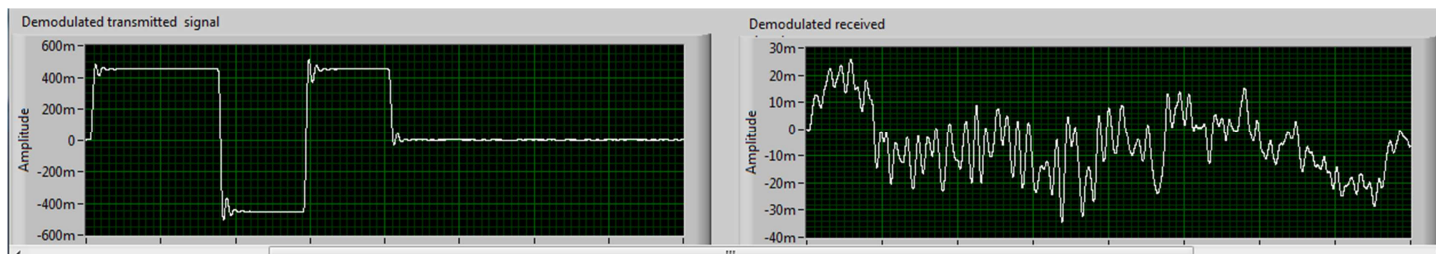

Figure 33. Example of time-domain waveform transmitted from EMAT to PZT.

\section{CONCLUSION}

In this work, the preliminary results of theoretical and experimental research activities toward the development of methods for transmission of information by acoustic means along metal infrastructures were discussed. Based on the initial reviews of the metal piping infrastructure in a commercial nuclear power plant, the CVCS piping was identified as a potential candidate for acoustic communications medium. Numerical modeling using COMSOL $^{\mathrm{TM}}$ multi-physics software tool was conducted to better understand dependence of performance (bandwidth and attenuation) on parameters such as waveform type, transducer type, and transmission medium. In parallel, a benchtop system was assembled for performing experimental evaluations. Based on the results of experimental activities to date a number of parameters that could affect the transmission of signals over long distances were identified. The focus of experimental activities reported here was on assessing the adoption of transducers based on both the piezoelectric and the EMAT technology. In addition, preliminary evaluations were conducted of frequency modulation (FM) and on-off keying (OOK) modulation to determine the optimal modulation scheme for transmission of signal under the constraint of limited signal generator power. Based on preliminary results of signal transmission, OOK looks like the preferred modulation scheme for information transmission. Information transmission by means of ASK and PSK schemes will be further explored in the next quarter. Benchmarks for information transmission rate, signal to noise ratio, and bit error rate will be established. The studies will consider tradeoffs between signal to noise ratio of received signals and information transfer rates. 


\section{ACKNOWLEDGEMENTS}

The authors acknowledge the contribution of D. Ozevin and L. Zhang (University of Illinois at Chicago).

\section{REFERENCE}

1. S. Bakhtiari, P. Ramuhalli, D.L. Brenchley, Argonne National Laboratory, unpublished information (2012).

2. A. Bari and J. Jiang, "Deployment strategies for wireless sensor networks in nuclear power plants," Nuclear Technology 187, 82 (2014).

3. C.D. Taylor et al., "Measurement of RF propagation into concrete structures over the frequency range $100 \mathrm{MHz}$ to $3 \mathrm{GHz}$," Wireless Personal Communications. Springer US, 131-144 (1997).

4. T. Phillipidis and D.G. Aggelis, "Experimental study of wave dispersion and attenuation in concrete," Ultrasonics 43, 584-595 (2005).

5. G. Kokossalakis, "Acoustic data communication system for in-pipe wireless sensor networks," $\mathrm{PhD}$ thesis, Massachusetts Institute of Technology (2006).

6. H.W. Tomlinson, Jr., J.B. Deaton, Jr., E. Nieters, F. Ross, "Ultrasound communication system for metal structure and related methods," US Patent 7654148 B2 (2010).

7. M.B. Yeary, J.K. Antonio, T.D. Hosman, "Ultrasonic communication system for communication through RF-impervious enclosures and abutting structures," US Patent Application 20140119162 A1 (2014).

8. T. Palmer, D. Tegerdine, E. Bean, F.R. Llorente, "Wireless acoustic communications method and apparatus," US Patent Application WO2013102761 A2 (2013).

9. C. Bacher, P. Palensky, S. Mahlknecht, "Low cost data transmission via metallic solids for sensor networking," Emerging Technologies, 2005, Proceedings of IEEE Symposium on, 193-198 (2005).

10. Y. Jin, Y. Ying, D. Zhao, "Data communications using guided elastic waves by time reversal pulse position modulation: Experimental study," Sensors 13, 8352-8376 (2013).

11. S. Charkraborty, G.J. Saulnier, K.W. Wilt, E. Curt, H.A. Scarton, R.B, Litman, "Low-power, lowrate ultrasonic communications system transmitting axially along a cylindrical pipe using transverse waves," IEEE Trans. Ultrason. Ferroelectr. Freq. Control 62(10), 1788 (2015).

12. H. Sakuma, K. Nakamura, S. Ueha, "Two-way communication over gas pipe-line using multicarrier modulated sound waves with cyclic frequency shifting," Acoust. Sci. Tech. 27(4), 225 (2006).

13. T. Arikan, T.J. Riedl, A.C. Singer, J. Younce, "Comparison of OFDM and Single-Carrier Schemes for Doppler Tolerant Acoustic Communications," OCEANS 2015 (submitted).

14. T. Riedl and A.C. Singer, "Towards a video-capable wireless underwater modem: Doppler tolerant broadband acoustic communication," Underwater Communications and Networking (UComms), 1-5 (2014).

15. L.N. Rajan, M. Lu, N.R. Shanbhag, A.C. Singer, "BER-Optimal Analog-to-Digital Converters for Communication Links," IEEE Transactions on Signal Processing, 60(7), 3683-3691 (2012).

16. A.C. Singer, J.K. Nelson, S.S. Kozat, "Signal Processing for Underwater Acoustic Communications," IEEE Communications Magazine, 47(1), 90-96 (2009).

17. T. J. Riedl and A. C. Singer, "Broadband Doppler Compensation: Principles and New Results," Proceedings of the Forty-Fifth Asilomar Conference on Signals, Systems and Computers (2011).

18. T. Riedl and A. Singer, "Must-read: Multichannel Sample-by-sample Turbo Resampling Equalization and Decoding," Proceedings of 2013 MTS/IEEE OCEANS - Bergen, 1-5 (2013).

19. IAEA Safety Standards Series, Design of Reactor Containment Systems for Nuclear Power Plants, NS-G-1.10 (2004).

20. W. Sebrell, "The Potential for Containment Leak Paths Through Electrical Penetration Assemblies Under Sever Accident Conditions,” NUREG/CR-3234, SAND83-0538 (1983). 
21. M. H. Shackelford, T. R. Bump, R. W. Seidensticker, "Characterization of Nuclear Reactor Containment Penetrations Final Report," NUREG/CR-3855, SAND84-7180, ANL-84-87 (1985).

22. Westinghouse Technology Systems Manual, Section 5.6, Containment Penetrations and Isolation Systems, USNRC HRTD Rev. 0408.

23. B.S. Lee, R. Travis, E. Grove, A. Di Biasio, "Aging Assessment of Westinghouse PWR and General Electric BWR Containment Isolation Functions," NUREG/CR-6339, BNL-NUREG-52462, Brookhaven National Laboratory (1996).

24. Westinghouse Electric Company, "The Westinghouse Pressurized Water Reactor Nuclear Power Plant," Water Reactor Divisions (1984).

25. Westinghouse Technology Systems Manual, Section 4.1, Chemical and Volume Control System, USNRC HRTD Rev. 1208 and Rev 0598.

26. USNRC Technical Training Center, Pressurized Water Reactor (PWR) Systems, Reactor Concept Manual.

27. Braidwood Updated Final Safety Analysis Report, Revision 15 (2014).

28. R. Kolbe and E. Gahan, "Survey of Insulation Used in Nuclear Power Plants and the Potential for Debris Generation,"NUREG/CR-2403, SAND82-0927 Supplement No.1 (1982).

29. L. Tang and B. Wu, "Excitation Mechanism of Flexural-Guided Wave Modes F(1,2) and F(1,3) in Pipes," Journal of Nondestructive Evaluation, 36-59 (2017).

30. A. Demma, "The Interaction of Guided Waves with Discontinuities in Structures," Ph.D. Dissertation, Imperial College (2003).

31. J.L. Rose, "Ultrasonic guided waves in solid media," Cambridge University Press (2014).

32. M.G. Silk and K.F. Bainton, "The propagation in metal tubing of ultrasonic wave modes equivalent to Lamb waves," Ultrasonics, 17(1), 11-19 (1979).

33. J.L. Rose, D. Jiao, J. Spanner, "Ultrasonic Guided Wave NDE for Piping," Materials Evaluation, 1310-1313 (1996).

34. J. Li and J.L. Rose, "Natural Beam Focusing of Non-axisymmetric Guided Waves in Large-diameter Pipes," Ultrasonics, 44, 35-45 (2006).

35. M.J.S. Lowe, D.N. Alleyne, P. Cawley, "Defect Detection in Pipes using Guided Waves," Ultrasonics, 36, 147-154 (1998).

36. C. Pei, S. Zhao, P. Xiao, Z. Chen, "A modified meander-line-coil EMAT design for signal amplitude enhancement," Sensors and Actuators A: Physical, 247, 539-546 (2016).

37. COMSOL Multiphysics R 4.1, User's Guide, COMSOL Inc. (2010).

38. S. Choi, H. Cho, C.J. Lissenden, "Selection of shear horizontal wave transducers for robotic nondestructive inspection in harsh environments," Sensors, 17(1), 5 (2017).

39. H. Nishino, T. Tanaka, S. Katashima, K. Yoshida, "Experimental investigation of mode conversions of the $\mathrm{T}(0,1)$ mode guided wave propagating in an elbow pipe," Japanese Journal of Applied Physics, 50, 046601 (2011).

40. A. Heifetz, S. Bakhtiari, X. Huang, S. Passerini, R.B. Vilim, "Acoustic communications at a nuclear facility using guided elastic waves on pipes," presented at Annual Review of Progress in Quantitative Nondestructive Evaluation (QNDE), Provo, UT, July 2017. 


\section{Argonne}

\section{Nuclear Engineering Division}

Argonne National Laboratory

9700 South Cass Avenue, Bldg. 208

Argonne, IL 60439

www.anl.gov 